\title{
TorsionNet: A Deep Neural Network to Rapidly Predict Small Molecule Torsion Energy Profiles with the Accuracy of Quantum Mechanics
}

Brajesh K. Rai", , Vishnu Sresht', Qingyi Yang², Ray Unwalla², Meihua Tu², Alan M. Mathiowetz ${ }^{2}$, and Gregory A. Bakken ${ }^{3}$

${ }^{1}$ Simulation and Modeling Sciences and ${ }^{2}$ Medicine Design, Pfizer Worldwide Research Development and Medical, 610 Main Street, Cambridge, Massachusetts 02139, United States

${ }^{3}$ Digital, Pfizer, Eastern Point Road, Groton, Connecticut 06340, United States

\begin{abstract}
Fast and accurate assessment of small molecule dihedral energetics is crucial for molecular design and optimization in medicinal chemistry. Yet, accurate prediction of torsion energy profiles remains challenging as the current molecular mechanics (MM) methods are limited by insufficient coverage of druglike chemical space and accurate quantum mechanical (QM) methods are too expensive. To address this limitation, we introduce TorsionNet, a deep neural
\end{abstract}


network (DNN) model specifically developed to predict small molecule torsion energy profiles with QM-level accuracy. We applied active learning to identify nearly 50k fragments (with elements $\mathrm{H}, \mathrm{C}, \mathrm{N}, \mathrm{O}, \mathrm{F}, \mathrm{S}$, and $\mathrm{Cl}$ ) that maximized the coverage of our corporate compound library and leveraged massively parallel cloud computing resources for DFT torsion scan of these fragments, generating a training dataset of 1.2 million DFT energies. After training TorsionNet on this dataset, we obtain a model that can rapidly predict the torsion energy profile of typical druglike fragments with DFT-level accuracy. Importantly, our method also provides an uncertainty estimate for the predicted profiles without any additional calculations. In this report, we show that TorsionNet can accurately identify the preferred dihedral geometries observed in crystal structures. Our TorsionNet-based analysis of a diverse set of protein-ligand complexes with measured binding affinity shows a strong association between weak bioactivity and high ligand strain and low potency. We also present practical applications of TorsionNet that demonstrate how consideration of DNN-based strain energy leads to substantial improvement in existing lead discovery and design workflows. TorsionNet500, a benchmark dataset comprising 500 chemically diverse fragments with DFT torsion profiles (12k MM and DFT-optimized geometries and energies), has been created and is made publicly available. 


\section{Chemical Environment Representation using Symmetry Function}

The local atomic environment around a given rotatable bond is represented by a fixed-length numerical vector, using a set of symmetry functions $\left\{G_{r}^{\alpha}, G_{\theta}^{\alpha \beta}, G_{\varphi}^{\alpha \beta}\right\}$ that are invariant not only to translation and rotation of the whole molecule but also to the permutation of atoms that are of the same type. Symmetry functions $G_{r}^{\alpha}$ and $G_{\theta}^{\alpha \beta}$ were first proposed by Behler and Parrinello ${ }^{1}$ to describe the radial and angular distributions, respectively, of the atoms in the neighborhood of a reference center. In this work, we extended the original Behler-Parrinello method by introducing torsion symmetry functions $\left(G_{\varphi}^{\alpha \beta}\right)$, which provides a more detailed description of the local dihedral environment that determines the torsion energy profile. Here, $\alpha$ and $\beta$ represent one of the 9 atom types, one for each of the chemical elements $\mathrm{H}, \mathrm{C}, \mathrm{N}, \mathrm{O}, \mathrm{F}, \mathrm{S}$, and $\mathrm{Cl}$, and 2 additional types for atoms with negative and positive formal charge. 
A common component of all three symmetry functions is the cutoff function $f_{c}$, which defines the chemical environment around a given reference center

$f_{c}\left(R_{i j}^{\alpha}\right)=\left\{\begin{array}{cc}0.5 \cdot\left[\cos \left(\frac{\pi R_{i j}^{\alpha}}{R_{c}}\right)+1\right] & \text { for } R_{i j}^{\alpha} \leq R_{c} \\ 0.0 & \text { for } R_{i j}^{\alpha}>R_{c}\end{array}\right.$

where $R_{i j}^{\alpha}$ is the distance between the reference center $i$ and the neighboring atom $j$ of type $\alpha$ with in the cutoff radius $R_{c}$.

Radial symmetry functions use a Gaussian-weighted sum of cutoff functions, $f_{c}$, to describe radial arrangement of neighboring atoms of type $\alpha$ around the reference center,

$G_{r}^{\alpha}=Z_{b} Z_{c} \sum_{j=1}^{N} e^{-\eta\left(R_{i j}^{\alpha}-R_{s}\right)^{2}} \cdot f_{c}\left(R_{i j}^{\alpha}\right)$

Here, $Z_{b}$ and $Z_{c}$ represent the atomic number of the two atoms that define the specified rotatable bond and the reference center $i$ was chosen as the mid-point of that bond. Parameters $\eta$ and $R_{S}$, which represent the width and shift of the Gaussians were set to $10^{-4}$ and 0 , respectively. The radial distribution of each atom of type $\alpha$ around the reference center $i$ was generated using seven different cutoff radii $\left(R_{c}=1.5,2.0,2.5,3.0,4.0,6.0\right.$, and $\left.10.0 \AA\right)$, yielding 63 radial symmetry function elements ( 7 cutoff radii for each of the 9 atom types). 
Angular symmetry functions depend on the angle $\theta_{i j k}$, centered at $i$, and enclosed by vectors $R_{i j}^{\alpha}$ and $R_{i k}^{\beta}$ that connect atoms $j$ and $k$ of type $\alpha$ and $\beta$, respectively, to the reference center. The sum over all cosines with respect to any neighbor $j$ and $k$, each of which is weighted by a Gaussian of the three interatomic distances and the respective cutoff functions were calculated as $G_{\theta}^{\alpha \beta}=2^{1-\zeta} Z_{b} Z_{c} \sum_{j} \sum_{k \neq j}\left[\left(1+\lambda \cdot \cos \theta_{i j k}\right)^{\zeta} \cdot e^{-\eta\left(R_{i j}^{2}+R_{i k}^{2}+R_{j k}^{2}\right)} \cdot f_{c}\left(R_{i j}^{\alpha}\right) \cdot f_{c}\left(R_{i k}^{\beta}\right) \cdot f_{c}\left(R_{j k}^{\alpha \beta}\right)\right]$.

As in the case of the radial symmetry functions, $Z_{b}$ and $Z_{c}$ represent the atomic number of the two atoms that define the specified rotatable bond and the reference center $i$ was chosen as the mid-point of that bond. Parameters $\zeta, \lambda$, and $\eta$ were set to $0.5,0.5$, and $10^{-4}$, respectively. In order to keep the number of angular symmetry function elements small, only a single cutoff radius, $R_{c}=4.0 \AA$, was used. With nine atom types and one fixed set of parameters, our angular symmetry functions were described by 45 elements (number of unique atom-type pairs).

Torsion symmetry functions were defined by dihedral angles $\varphi_{i j k l}$ that includes atoms $j$ and $k$ of the specified rotatable bond. Pairs $i$ and $l$ are any atoms of type $\alpha$ and $\beta$, respectively, but located on opposite side of the rotatable bond $(j k)$.

$G_{\varphi}^{\alpha \beta}=2^{1-\zeta} Z_{b} Z_{c} \sum_{j} \sum_{k \neq j}\left[\left(1+\lambda \cdot \cos \varphi_{i j k l}\right)^{\zeta} \cdot e^{-\eta\left(R_{i j}^{2}+R_{k l}^{2}+R_{i l}^{2}\right)} \cdot f_{c}\left(R_{i j}^{\alpha}\right) \cdot f_{c}\left(R_{k l}^{\beta}\right) \cdot f_{c}\left(R_{i l}^{\alpha \beta}\right)\right]$. 
All parameters of the torsion symmetry function were identical to those we used for the angular component. However, to construct a more detailed representation of the dihedral environment, torsion symmetry functions were calculated using a range of cutoff radii $\left(R_{c}=2.5,3.5,5.0\right.$, and $10.0 \AA$ A), generating 180 values for this component.

In addition to symmetry functions, we used five additional features that describe the geometric and chemical characteristics of the specified torsion. These include the dihedral angle, the length of the central rotatable bond, the distance between the two terminal atoms, and the product of the atomic numbers of the two central and the two terminal atoms. These additional features were combined with the radial, angular, and torsion symmetry function elements to produce a 293dimensional atomic environment vector (AEV) that encodes the chemical environment around a given rotatable bond.

The key features of these symmetry functions include their smoothness to minor perturbation in geometries and their invariance to translation, rotation, and permutation, making these functions important and well-received descriptors for machine learning methods ${ }^{2}$.

\section{Deep Neural Network}

As in the case of the Random Forest, DNN regression models were also trained using AEVs as the input features and the corresponding relative energies as the target. However, unlike RF, each 
input feature to the DNN model was first standardized by subtracting the mean and scaling to unit variance. The model architecture was defined using the Keras deep learning framework (version 2.2.0, http://keras.io) with the TensorFlow (version 1.7.0, www.tensorflow.org) backend. The network is comprised of 5 fully connected (FC) hidden layers, with 2930 nodes (corresponding to $10 p$, where $p$ is the number of input features) in the first hidden layer. Each subsequent hidden layer contained a decreasing number of nodes, $5 p, 3 p, 2 p, 1 p$, respectively. The output of each FC layer was transformed by the ReLU activation function, followed by Batch Normalization and a drop out layer with a fixed dropout value of 0.2. The network was trained using $70 \%$ of the torsional profile data, with $10 \%$ of the DFT data held out for validation; the remaining $20 \%$ of the dataset was used as the test set. Weights in each layer were initialized using the default Glorot scheme. The network was optimized for logcosh loss using Adam with a fixed learning rate of $10^{-4}$ and default settings for other optimizer parameters $\left(\beta_{1}=0.99, \beta_{2}=0.999\right)$. Models were trained with a batch size of 256 for 5000 epochs. Since no early stopping criteria were applied, each model was trained for a full 5000 epochs, while saving the best model (with the smallest validation loss).

Hyperparameter tuning was performed by exploring the ranges of values for the learning rate, batch size, and drop out that are commonly used when training deep neural networks. We also tuned the width and the depth of the network, testing architectures that contained between 2 and 6 [FC-ReLU-BN-dropout] blocks. The learning rate (with Adam optimizer) was varied from 1e3 to 1 e- 6 with 10-fold increments. The batch size was sampled from one of the following values: 
$64,128,256,512,1024,2048,4096$, and 8192. Drop out values of $0.05,0.1,0.2,0.3$, and 0.5 were explored, while keeping the same dropout rate for each layer.

\section{TorsionNet500 fragments}

The Supplementary Information file TorsionNet500.mol contains a single molecular mechanics structure for each of the 500 distinct fragments in the TorsionNet500 benchmark set. Each molecule in this file has the following associated SD properties: 1) Torsion_atoms specifies the atomic indices of the four torsion atoms that were used to generate the torsion scan profile, 2) DFT_energy_profile and TorsionNet_energy_profile contain the relative energy of the intermediate conformations based on DFT and TorsionNet, respectively (comma-separated list of energy@angle values).

The Supplementary Information file TorsionNet500_qm_opt_geometries.mol contains 12k DFToptimized structures of the TorsionNet500 benchmark set, respectively. For each fragment in this set, geometry and energy of 24 intermediate conformations are included. The SD properties attached to each molecule in these sets specify the torsion atom indices (Torsion_atoms), the corresponding torsion angle (Torsion_angle), and the DFT energy of that conformation (Energy) 
The Supplementary Information file TorsionNet500_mm_opt_geometries.mol contains 12k MMoptimized structures of the TorsionNet500 benchmark set, respectively. For each fragment in this set, geometry and energy of 24 intermediate conformations are included. The SD properties attached to each molecule in these sets specify the torsion atom indices (Torsion_atoms), the corresponding torsion angle (Torsion_angle), the MMFF energy of that conformation $($ Energy_ $M M)$, and the DFT energy of that conformation $\left(\right.$ Energy_$\left._{-} Q M\right)$

Note 1: Physchem properties were calculated using MolProp Python toolkit from OpenEye (release 2.5.3, OpenEye Scientific Software, Santa Fe, NM). OEGetHBondDonorCount and OEGetHBondAcceptorCount functions were used to calculate number of hydrogen bond donors and acceptors, respectively, based on the definition of Mills and Dean ${ }^{3}$. Fraction C sp3 were calculated using OEGetFractionCsp3 function, as described by Lovering et $\mathrm{al}^{4}$. $\log \mathrm{P}$ was calculated using $O E G e t X \log P$ function, which implements the XLogP algorithm from Wang et $\mathrm{al}^{5}$. 
Note 2: To prevent biasing our analysis due to few over-represented protein targets or families in the PDBbind, we chose the refined set, which comprised 4852 non-redundant proteins $(90 \%$ sequence identity), rather than the full PDBbind set. We further filtered refined set based on the following criteria: 1) molecules containing only $\mathrm{H}, \mathrm{C}, \mathrm{N}, \mathrm{O}, \mathrm{F}, \mathrm{S}$, or $\mathrm{Cl}$ atoms $(\mathrm{n}=4719), 2)$ number of rotatable bonds between 2 and $8(n=2107), 3)$ molecular weight between 350 and 700 $(n=865)$, and 4) neutral molecules with no formal charge centers $(n=385)$. Here, the numbers in parentheses indicate the count of protein-ligand complexes that remained after applying the filtering criteria at each step.

Note 3: An internal dataset comprising of 4 protein targets and associated compounds were used to assess whether strain correction based on TorsionNet can be used to improve hit rate of structure-based virtual screening. Targets from 4 different protein families, including GPCR, Kinase, PDE, and nuclear hormone receptors, were used in this study. We will refer to these targets as Target A, Target B, Target C, and Target D. 100 potent compounds from each of the 4 targets were selected from the internal compound collection. A common set of 100k confirmed inactives that were identified from previous high throughput screening campaigns against these targets were used as decoys. A common docking protocol was used to dock and score the docking library against each target. Compounds were docked using an internal docking program known as AgDock 6 . The top-scoring AgDock pose of each compound was scored in-place using Glide. This docking protocol was chosen since in our internal benchmark studies it has been shown to produce better hit rate in structure-based virtual screening. 


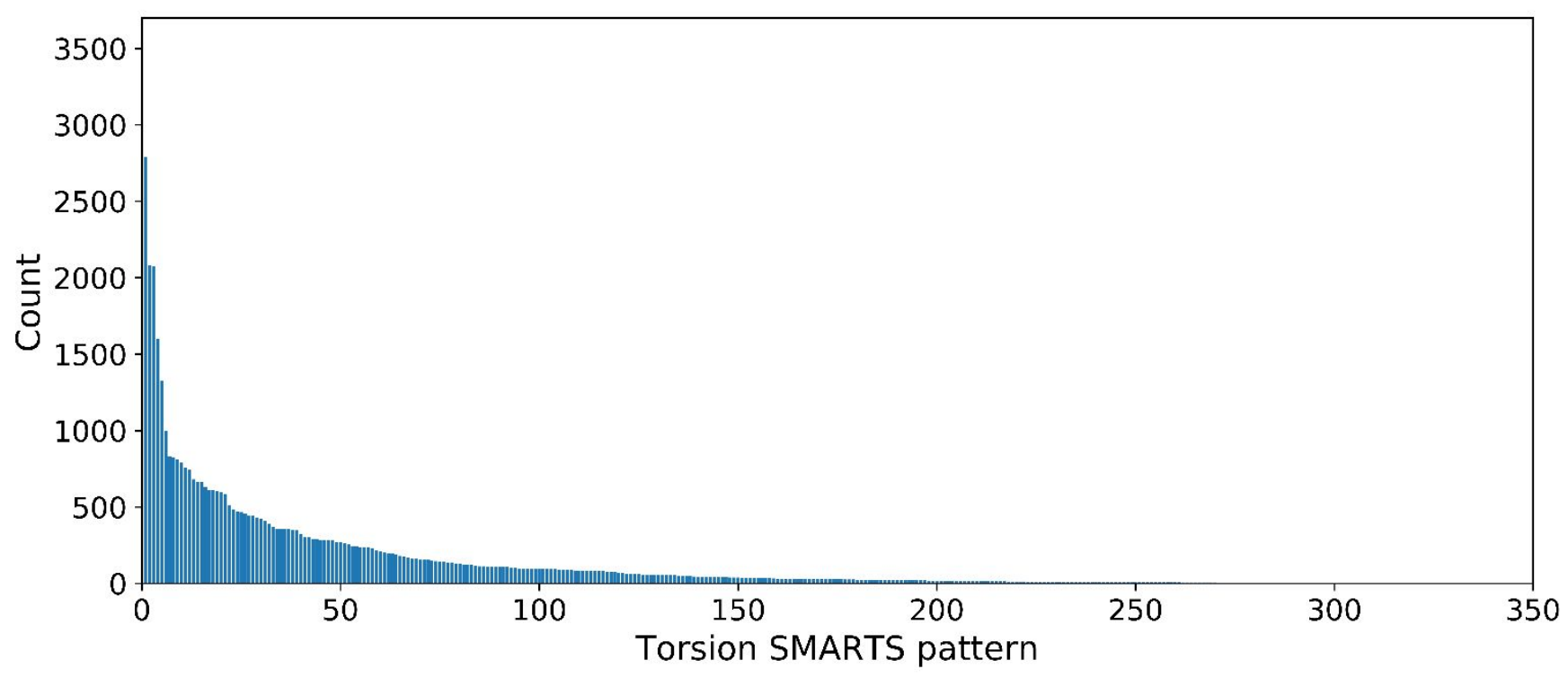

Figure S1. Distribution of torsion patterns ${ }^{7}$ present in the TorsionNet $50 \mathrm{k}$ training set. 

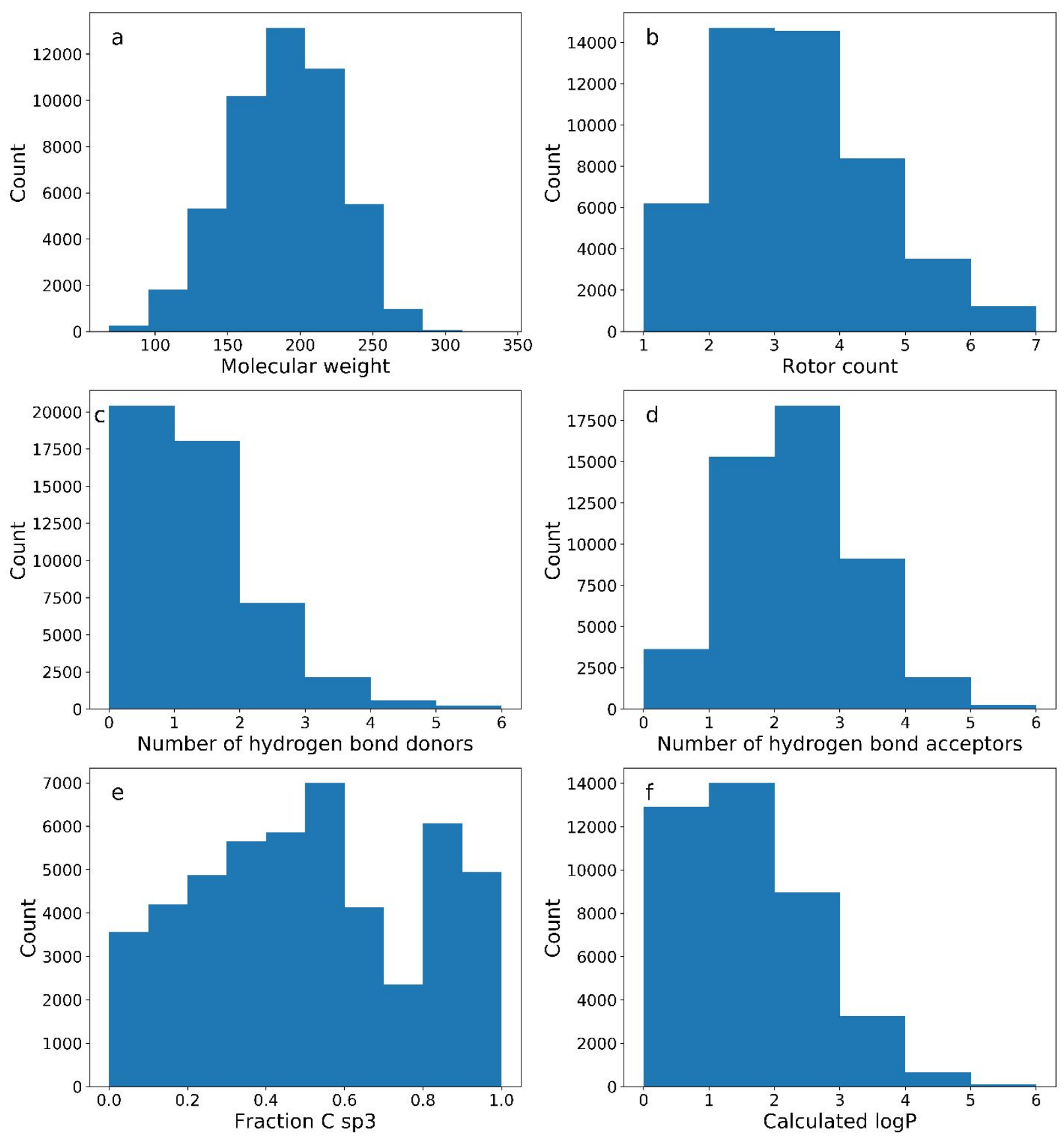

Figure S2. The key physicochemical properties of TorsionNet 50k training set fragments. (a) molecular weight. (b) rotatable bonds. (c) hydrogen bond donors. (d) hydrogen bond acceptors. (e) fraction sp3 carbons. (f) calculated $\log \mathrm{P}$. These properties were calculated using MolProp Python toolkit, as described in Note 1. 

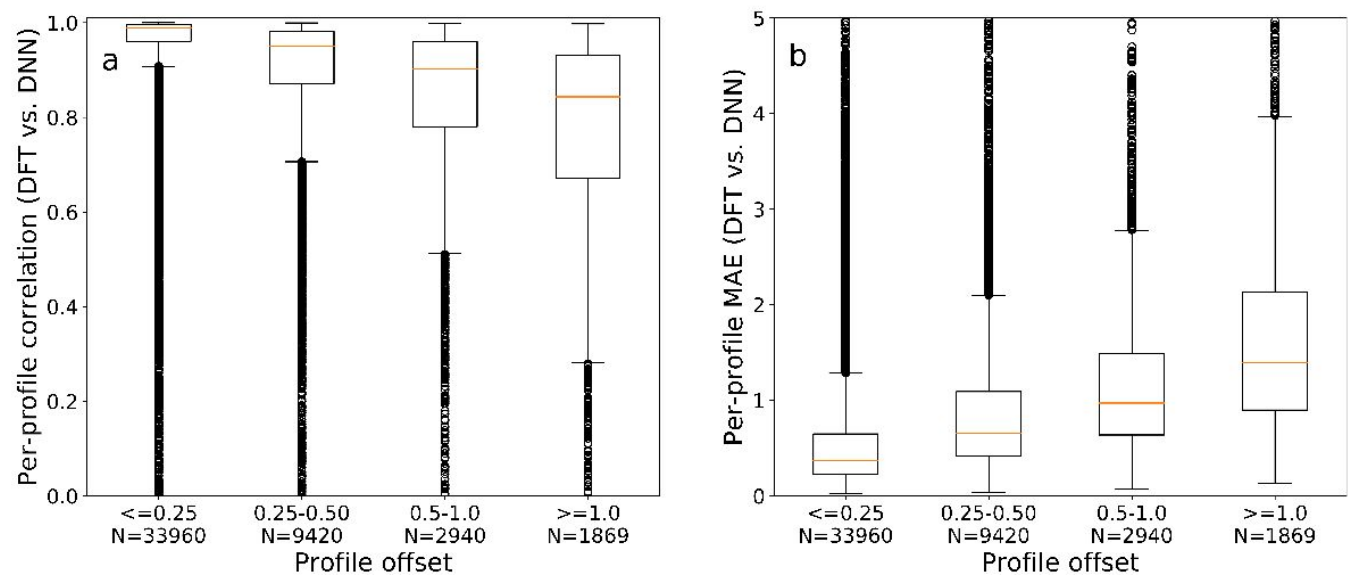

Figure S3. Distribution of Pearson correlation (a) and mean absolute error (b) of DNN test set profiles relative to DFT, binned by profile offset value. Number of fragments $(\mathrm{N})$ in each bin are shown as x-tick labels under each box. The horizontal line in each box represents the corresponding median value of the distribution. The whiskers extend to include the lowest and the highest data points within 1.5IQR (interquartile range) of the lower and upper quantile, respectively.
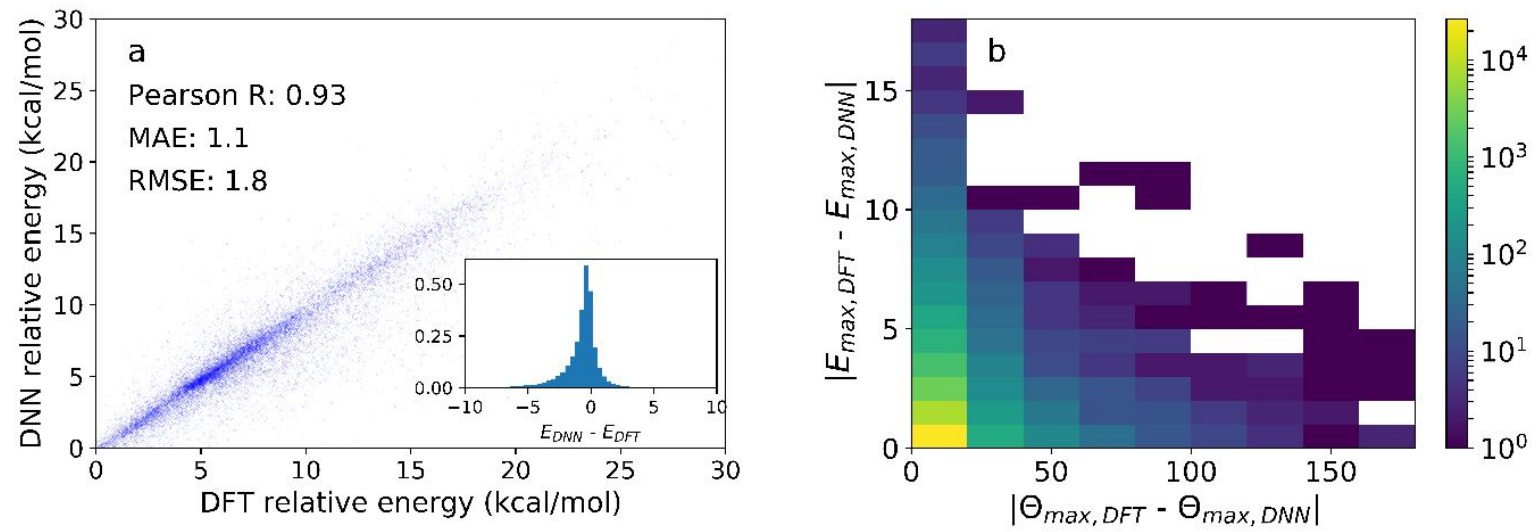

Figure S4. Performance of TorsionNet in predicting the highest energy peak using data from 50k fragments in the cross-validation test set. Relative energy of the highest peak in DFT profile vs. the relative energy of the nearest peak in the corresponding DNN profile (panel a). 2D Histogram of discrepancy in the position and energy of the highest peak in DFT profile compared to the nearest peak in the corresponding DNN profile (panel b). Inset in panel a shows normalized histogram of DFT vs DNN discrepancy. 

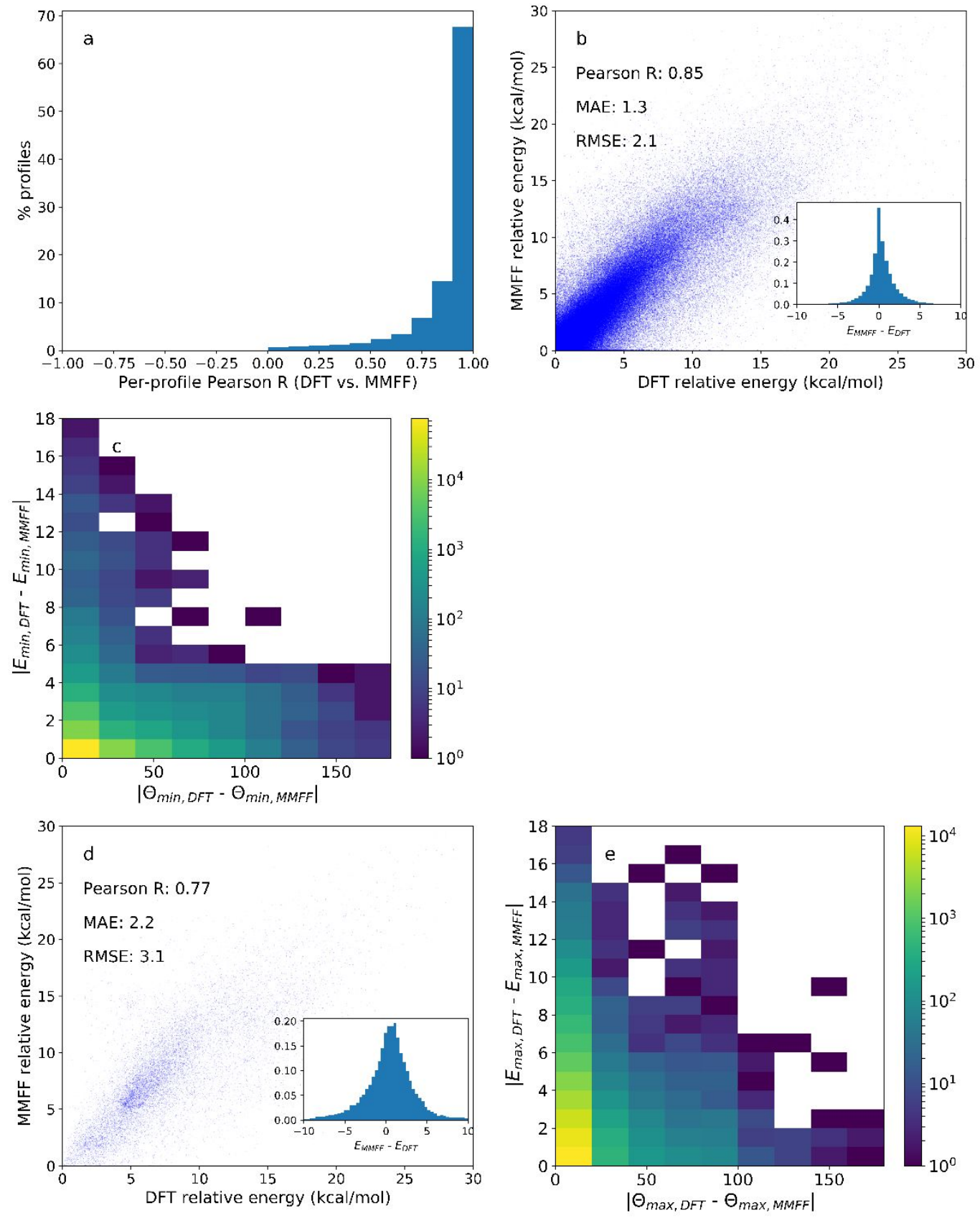
Figure S5. Histogram of per-profile Pearson correlation using torsion profiles from $50 \mathrm{~K}$ fragments: DFT vs. MMFF94s (panel a). Comparison of relative energies of 1.2 million intermediate conformers on torsion profiles: DFT vs. MMFF94s (panel b). 2D Histogram of MMFF94s prediction error in torsion angle (x-axis) and relative energy (y-axis) of 110k lowenergy minima in the test set profiles with respect to DFT (panel c). Relative energy of the highest peak in DFT profile vs. the relative energy of the nearest peak in the corresponding MMFF profile (panel d). 2D Histogram of discrepancy in the position and energy of the highest peak in DFT profile compared to the nearest peak in the corresponding MMFF profile (panel e). The 2D histograms are rendered using a logarithmic color scale according to the number of data points in each bin, yellow (largest) to purple (smallest). Relative energy of each intermediate conformation was calculated with respect to the lowest energy point on the corresponding profile. Inset in panel $b$ and $d$ shows normalized histogram of discrepancy of MMFF94s relative energies with respect to DFT.

\section{Comparison with MMFF}

As shown in Figure S5, for the entire set of 1.2 million relative energies from 50k profiles, MMFF achieves a Pearson correlation of 0.85 (vs. 0.94 for TorsionNet), MAE of $1.3 \mathrm{kcal} / \mathrm{mol}$

(vs. 0.7 for TorsionNet), and RMSE of $2.1 \mathrm{kcal} / \mathrm{mol}$ (vs. 1.2 for TorsionNet). Furthermore, $68 \%$ of MMFF profiles have Pearson correlation of $\geq 0.9$ relative to the corresponding DFT profiles (vs. $79 \%$ for TorsionNet). Nearly $69 \%$ of the local minima and $31 \%$ of the local high energy peaks in the MMFF profiles have matching minima or peak in the corresponding DFT profile such $|\Delta E| \leq 1 \mathrm{kcal} / \mathrm{mol}$ and $|\Delta \theta| \leq 20^{\circ}$ (vs. $84 \%$ and $64 \%$, respectively for TorsionNet). The Pearson correlation between the highest energy peak in DFT profiles to the nearest peak on the corresponding MMFF profile is 0.77 (vs. 0.93 for TorsionNet). 

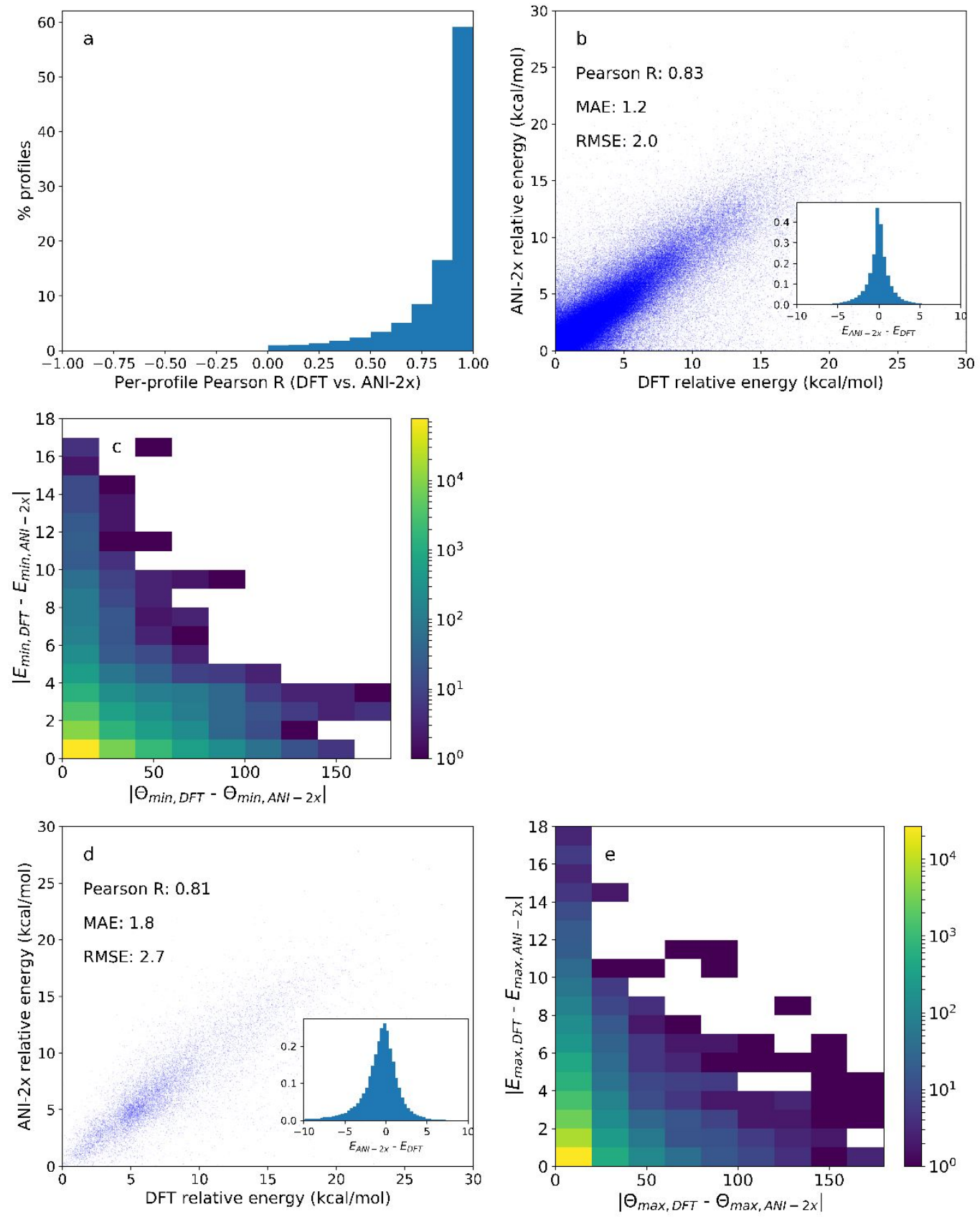
Figure S6. Histogram of per-profile Pearson correlation using torsion profiles from $50 \mathrm{~K}$ fragments: DFT vs. ANI-2x (panel a). Comparison of relative energies of 1.2 million intermediate conformers on torsion profiles: DFT vs. ANI-2x (panel b). 2D Histogram of ANI$2 \mathrm{x}$ prediction error in torsion angle (x-axis) and relative energy (y-axis) of $110 \mathrm{k}$ low-energy minima in the test set profiles with respect to DFT (panel c). Relative energy of the highest peak in DFT profile vs. the relative energy of the nearest peak in the corresponding ANI-2x profile (panel d). 2D Histogram of discrepancy in the position and energy of the highest peak in DFT profile compared to the nearest peak in the corresponding ANI-2x profile (panel e). The 2D histograms are rendered using a logarithmic color scale according to the number of data points in each bin, yellow (largest) to purple (smallest). Relative energy of each intermediate conformation was calculated with respect to the lowest energy point on the corresponding profile. Inset in panel $b$ and $d$ shows normalized histogram of discrepancy of ANI-2x relative energies with respect to DFT.

\section{Comparison with ANI-2x}

We compared how closely torsion energy profiles generated by this method matched those

calculated from DFT using the same fragments in our test set. Figure S6 shows a detailed comparison of ANI-2x relative to DFT. Distribution of per-profile Pearson correlation between ANI-2x and DFT (Figure S6a) is shifted to significantly lower values compared to the corresponding distribution shown for TorsionNet in Fig 3e. Whereas $82 \%$ of TorsionNet profiles have $\geq 0.9$ Pearson correlation to DFT, only $59 \%$ of ANI-2x profiles exceed this threshold. Comparison of the energies of intermediate torsion angle conformations show a similar trend: in comparison to ANI-2x (Figure S6b), TorsionNet shows significantly higher Pearson correlation (0.94 vs. 0.83 ) and smaller discrepancy (MAE of $0.7 \mathrm{kcal} / \mathrm{mol}$ for DNN vs. $1.2 \mathrm{kcal} / \mathrm{mol}$ for ANI-2x) with respect to DFT (main text Fig. 3f). 

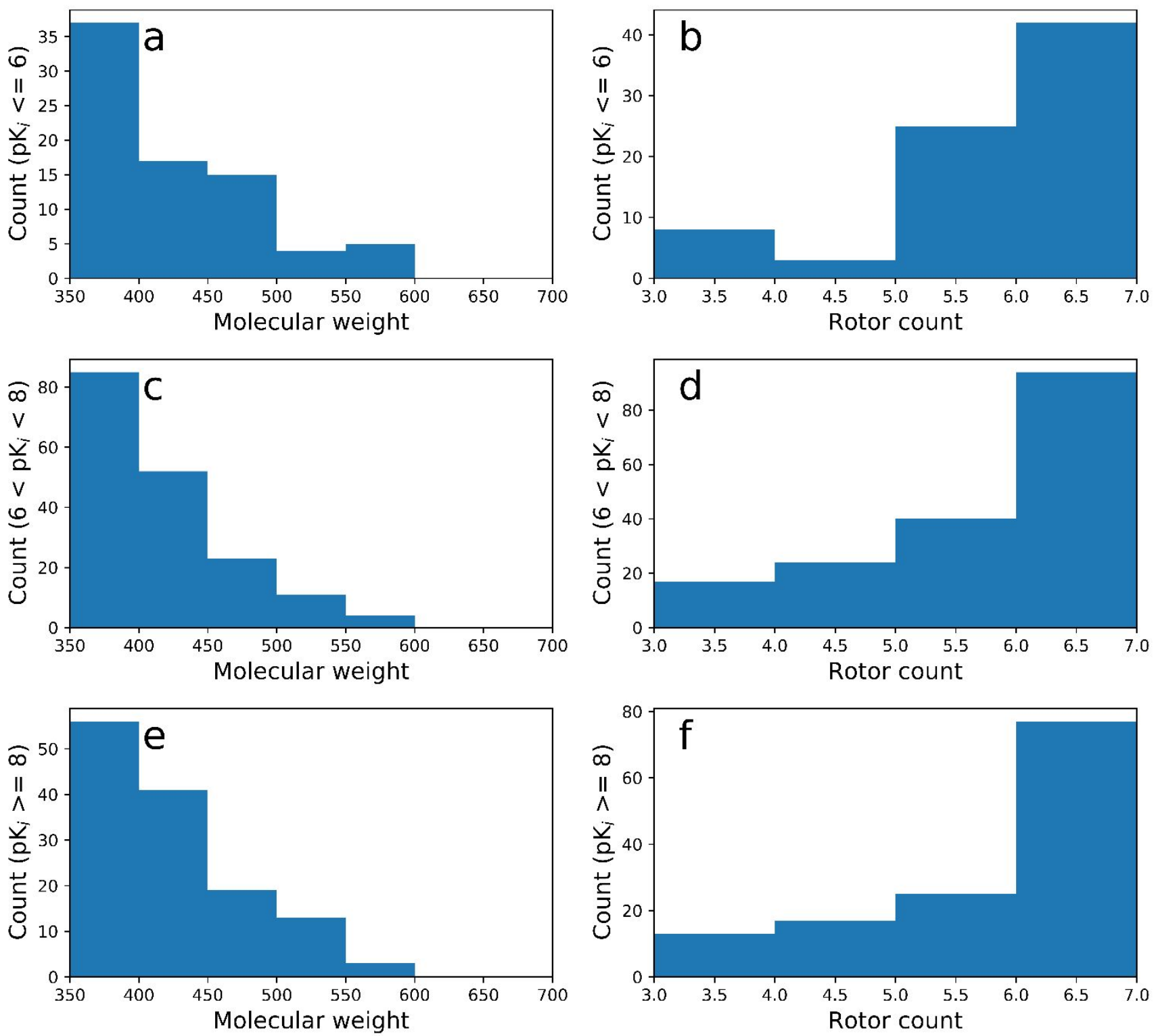

Figure S7. Distribution of molecular weight and rotor count of the ligands in the PDBbind refined subset. The ligands are split based on their measured binding affinity: low (pKi $<=6)$, middle $(6<\mathrm{pKi}<8)$, and high $(\mathrm{pKi}>=8)$. The ligands were extracted from PDBbind refined set using the filtering criteria as described in Note 2. 

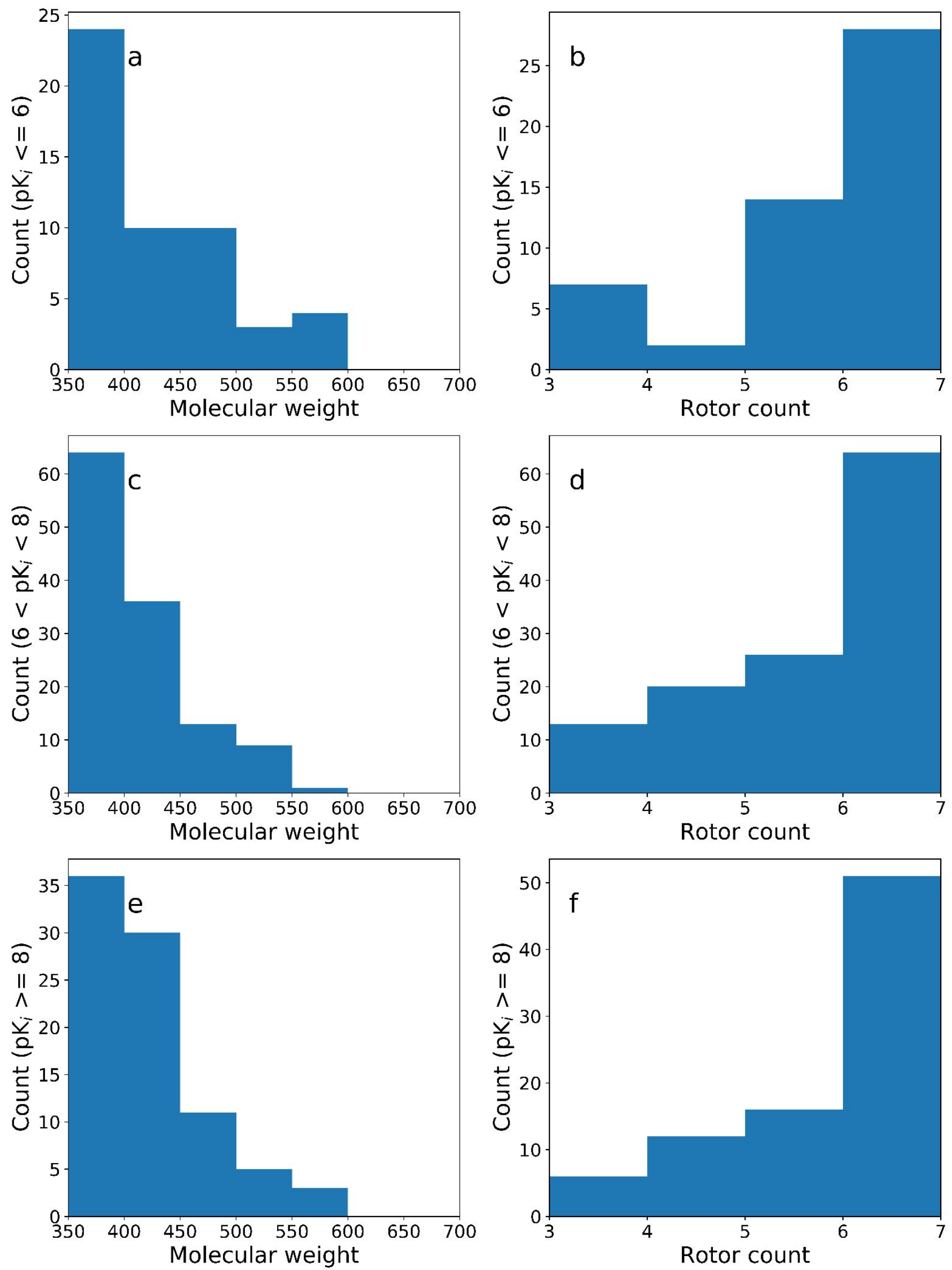
Figure S8. Distribution of molecular weight and rotor count of the ligands in the PDBbind refined subset containing higher resolution co-crystal structures. The ligands are split based on their measured binding affinity: low $(\mathrm{pKi}<=6)$, middle $(6<\mathrm{pKi}<8)$, and high $(\mathrm{pKi}>=8)$. The ligands were extracted from PDBbind refined set using the filtering criteria as described in Note 2. An additional filtering step based on the crystal structure resolution ( $2 \AA$ or better) was applied
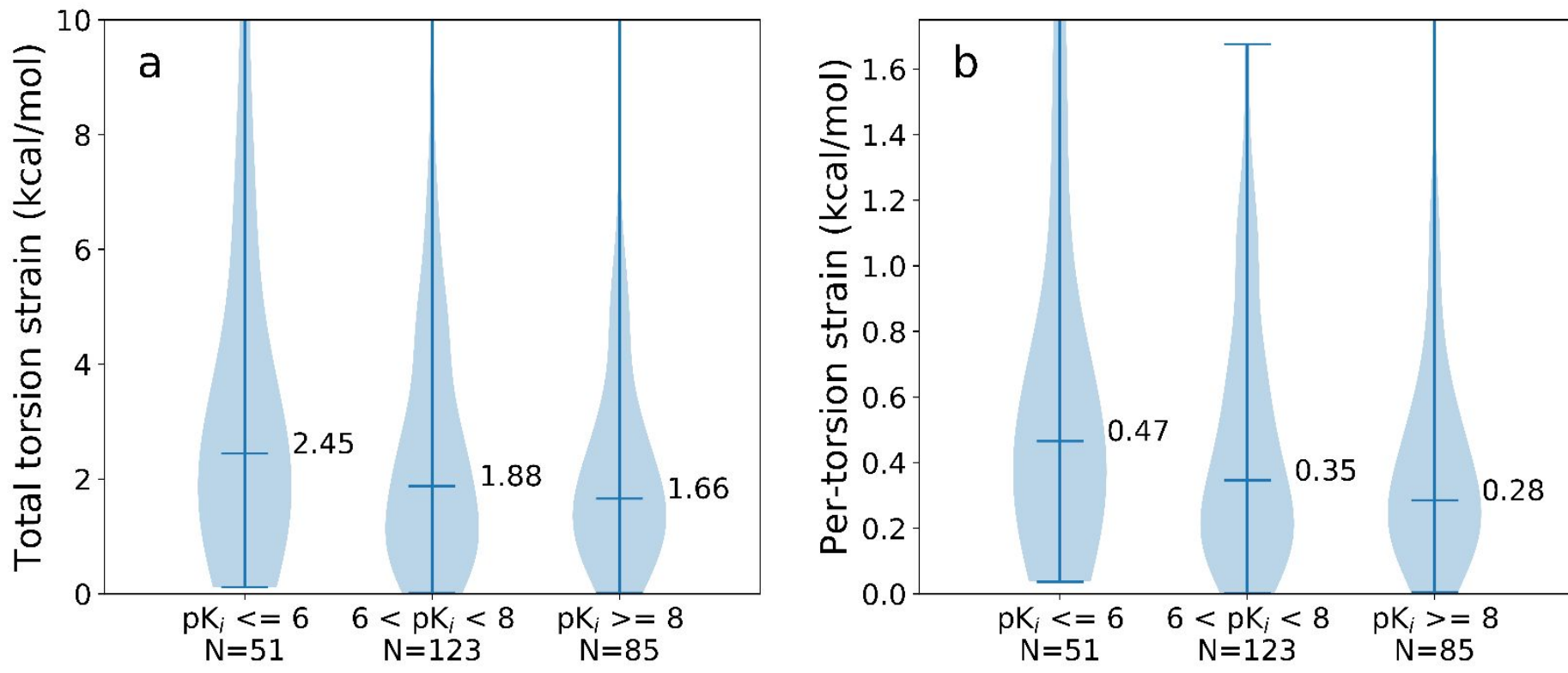

Figure S9. Distribution of ligand strain energy in a PDBbind refined subset containing higher resolution co-crystal structures: a) total torsion strain and b) per-torsion strain. The ligands were binned by their experimental binding affinity. Crystal structures used in this analysis were extracted from PDBbind refined set using the filtering criteria as described in Note 2. An additional filtering step based on the crystal structure resolution ( $2 \AA$ or better) was applied. 

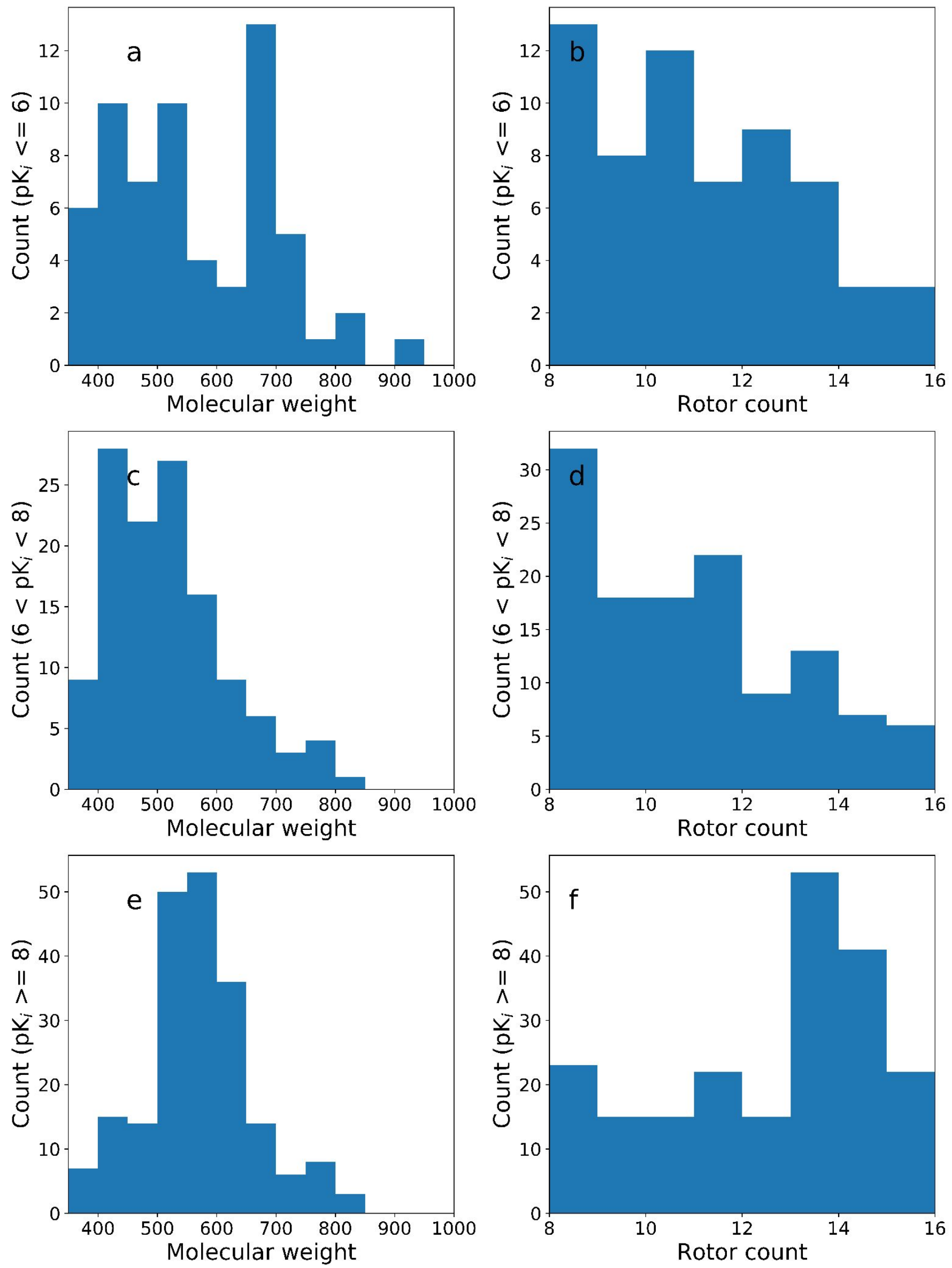
Figure S10. Distribution of molecular weight and rotor count of the molecules in the high conformational flexibility subset derived from PDBbind. The ligands are split based on their measured binding affinity: low $(\mathrm{pKi}<=6)$, middle $(6<\mathrm{pKi}<8)$, and high $(\mathrm{pKi}>=8)$. The ligands were extracted from the PDBbind refined set using the same filtering criteria as described in Note 2, except for the number of rotatable bonds and molecular weight. Only ligands with 8 to 15 rotatable bonds and molecular weight between 200 and 1000 were included in this subset.
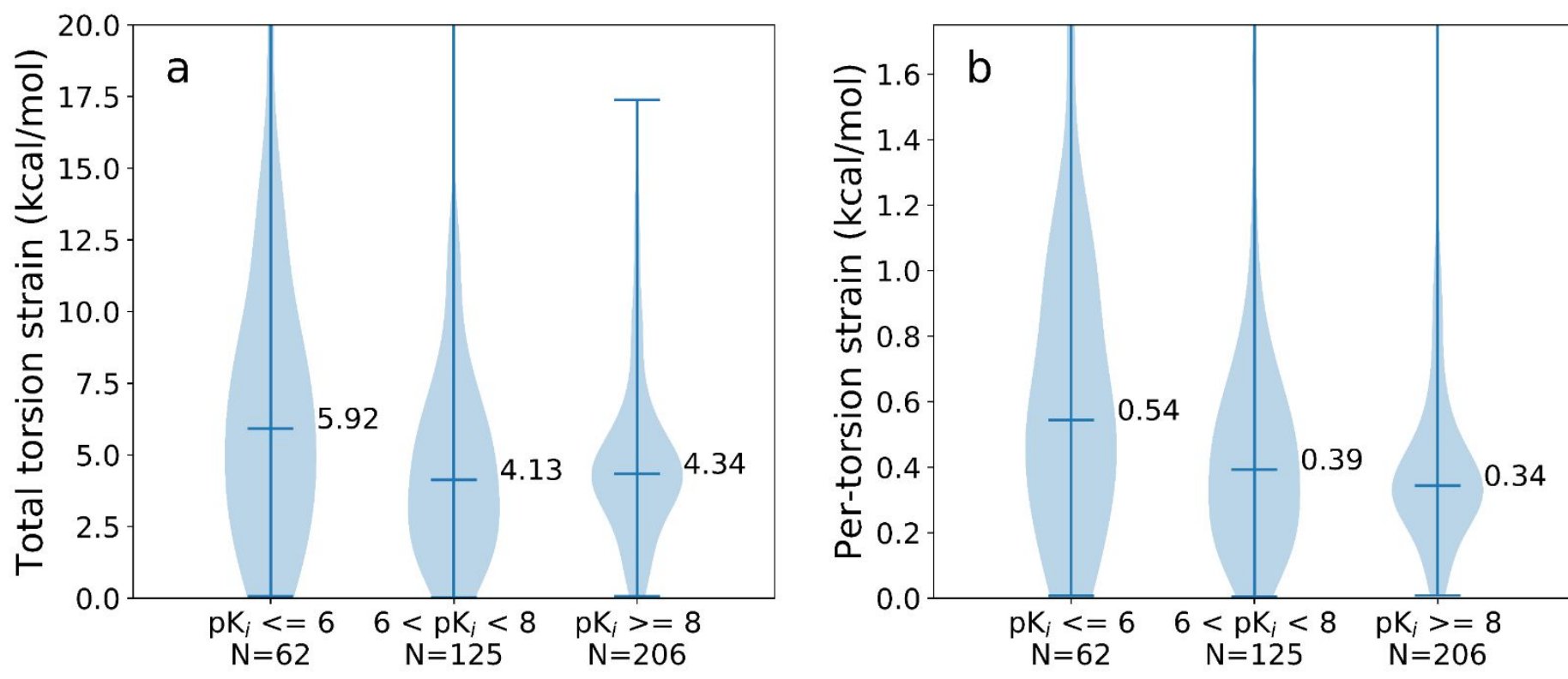

Figure S11. Distribution of ligand strain energy in a PDBbind refined subset containing ligands with high conformational flexibility: a) total torsion strain and b) per-torsion strain. The ligands were binned by their experimental binding affinity. Crystal structures used in this analysis were extracted from the PDBbind refined set using the same filtering criteria as described in Note 2, except for the number of rotatable bonds and molecular weight. Only ligands with 8 to 15 rotatable bonds and molecular weight between 200 and 1000 were included in this subset. 

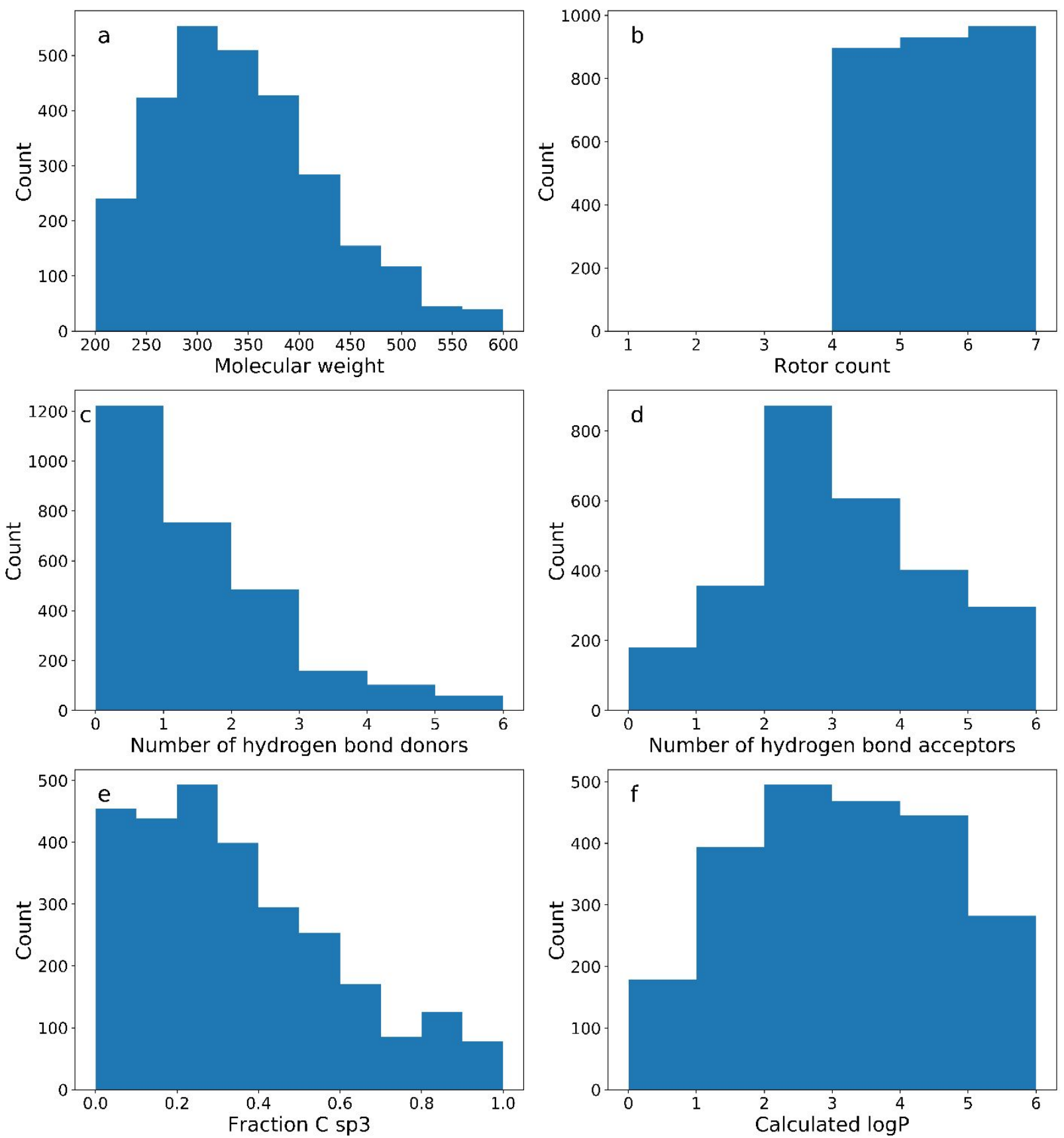

Figure S12. Key physicochemical properties of CSD set molecules used in conformation analysis. (a) molecular weight. (b) rotatable bonds. (c) hydrogen bond donors. (d) hydrogen bond acceptors. (d) fraction sp3 carbons. (f) calculated $\log$ P. These properties were calculated using MolProp Python toolkit, as described in Note 1. 

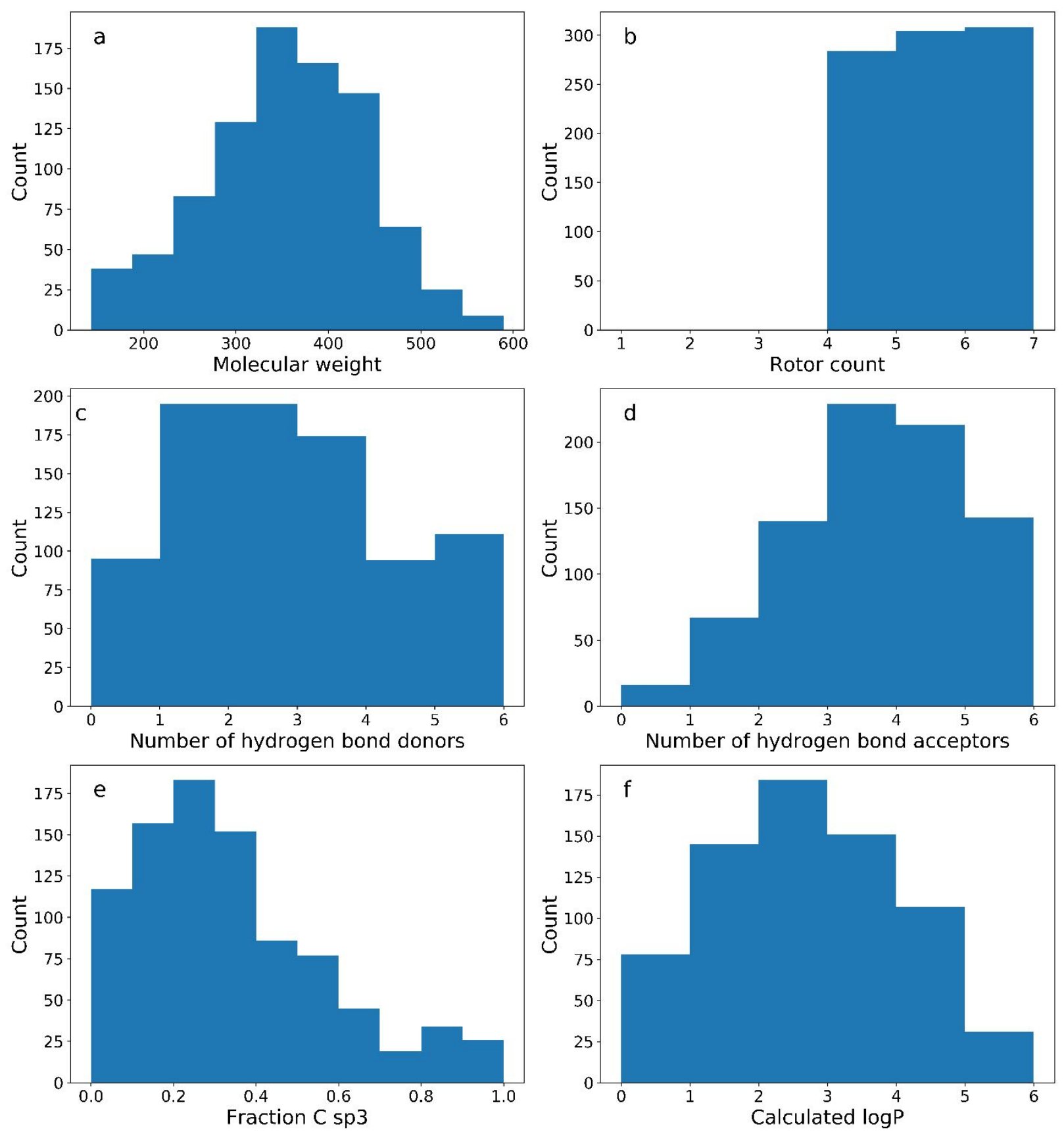

Figure S13. Key physicochemical properties of Platinum Diverse ${ }^{8}$ subset molecules used in conformation analysis. (a) molecular weight. (b) rotatable bonds. (c) hydrogen bond donors. (d) hydrogen bond acceptors. (e) fraction sp3 carbons. (f) calculated $\log \mathrm{P}$. These properties were calculated using MolProp Python toolkit, as described in Note 1. 

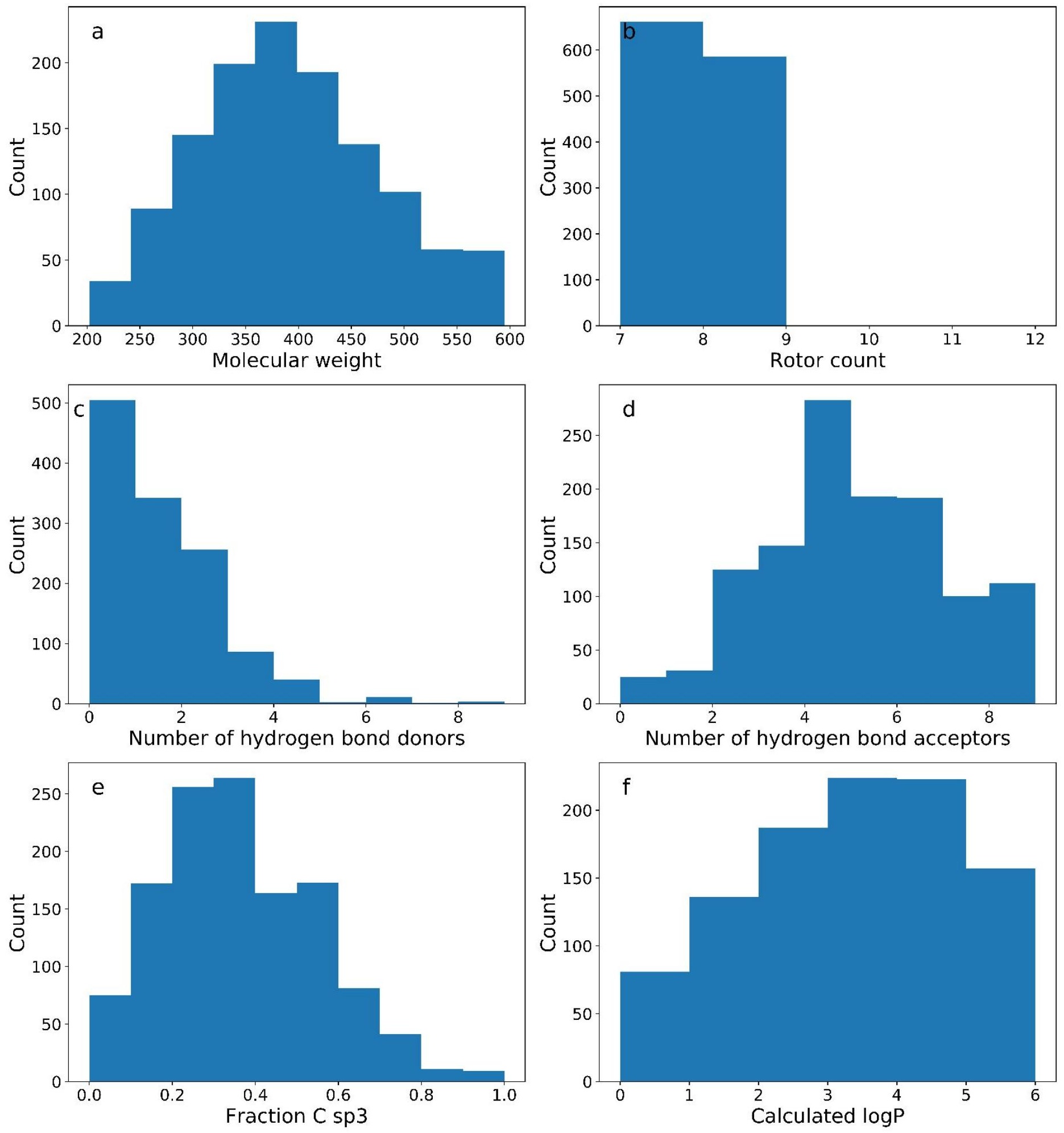

Figure S14. Key physicochemical properties of the CSD molecules in the high conformational flexibility subset. (a) molecular weight. (b) rotatable bonds. (c) hydrogen bond donors. (d) hydrogen bond acceptors. (d) fraction sp3 carbons. (f) calculated logP. 

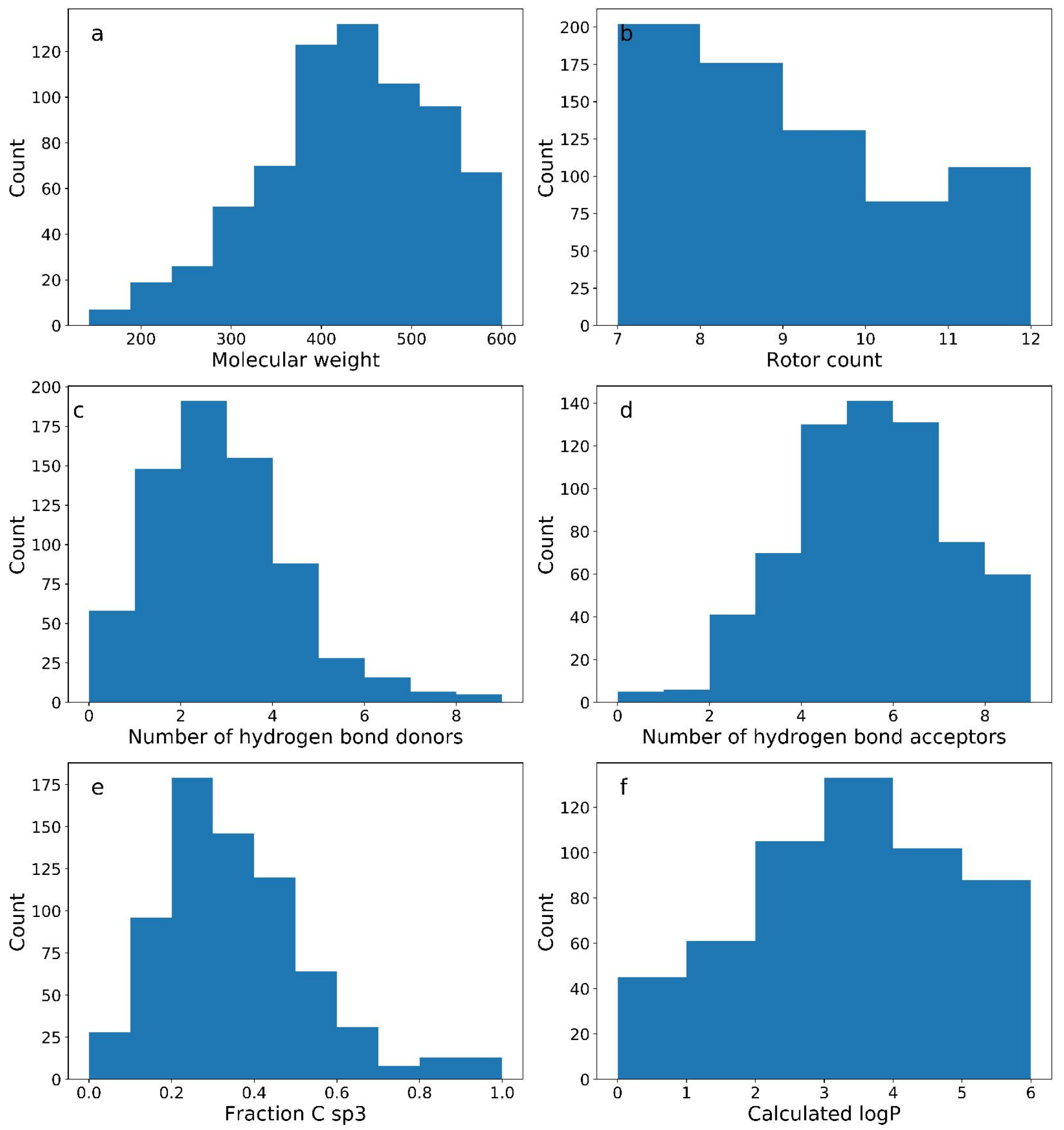

Figure S15. Key physicochemical properties of the molecules in the high conformational flexibility subset derived from the Platinum Diverse Dataset. (a) molecular weight. (b) rotatable 
bonds. (c) hydrogen bond donors. (d) hydrogen bond acceptors. (d) fraction sp3 carbons. (f) calculated $\log \mathrm{P}$.
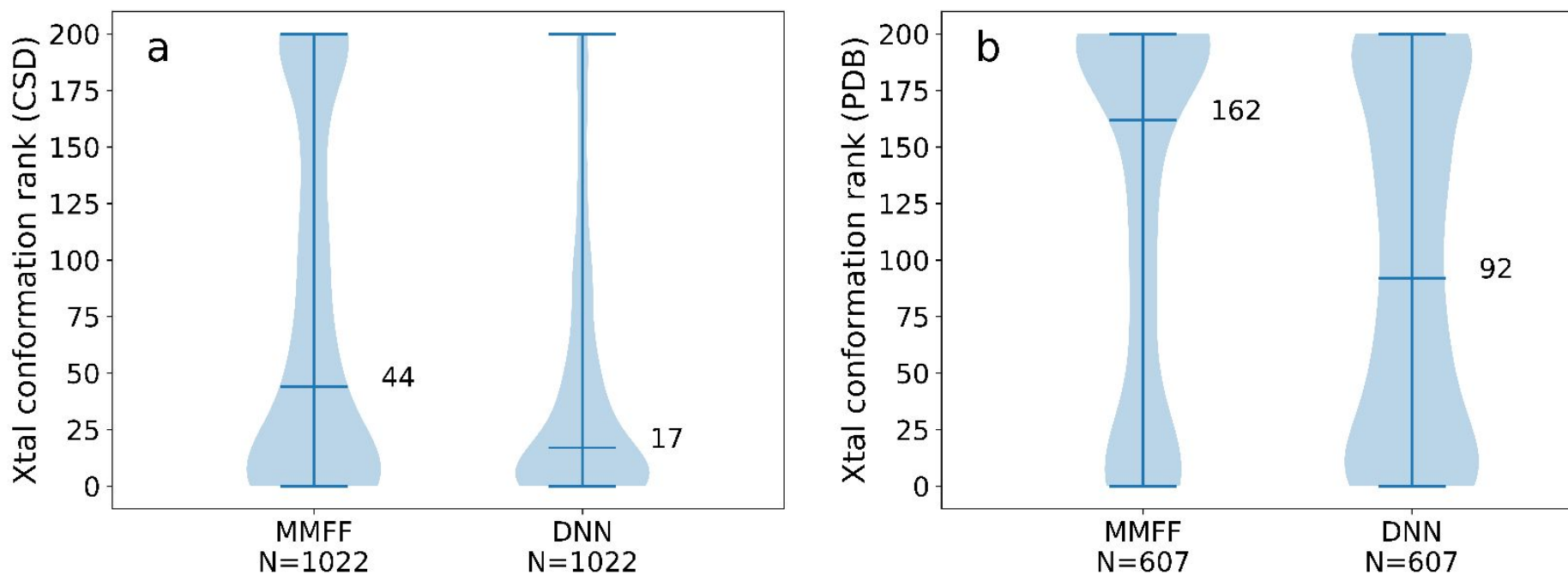

Figure S16. Testing the ability of TorsionNet to preferentially identify crystal structures from ensemble of low-energy conformations. Ranking of crystal structures seeded in conformation ensembles from (a) CSD and (b) PDB datasets comprising of high conformational flexibility molecules (CSD: number of rotatable bonds between 6 to 9; PDB: number of rotatable bonds between 6 to 13). For each test set structure, conformers and crystal structures were ranked using MMFF energy (excluding the stretch and angle bend components) and TorsionNet strain energy. Here, lower rank represents better performance.

Table S1: List of torsion patterns present in the TorsionNet500 benchmark. The definition of torsion patterns was adopted from Scharfer ${ }^{7}$. For each torsion pattern, a representative TorsionNet500 fragment along with the number of examples containing that pattern are shown.

SMARTS

Count 


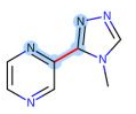

4

[a:1][c:2]!@[CX4H2:3][a:4]

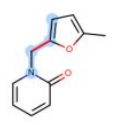

5

[a:1][ar6:2]!@[ar5:3][a:4]

12

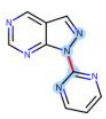

6

[c:1][CX4H2:2]!@[OX2:3][c:4]

10<smiles>[C]1CCCCC1</smiles>

7

[c:1][c:2]([cHO])!@[c:3][nX2H0:4]

9

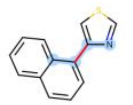

8

[c:1][c:2]!@[c:3][c:4]!@c

9

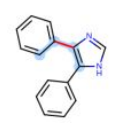

9

[cH1:1][c:2]([cH1])!@[NX3H1:3][C,c:4]

7 

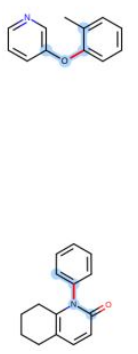

$$
3
$$
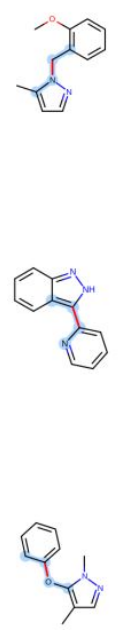
$19 \quad[\mathrm{c}: 1][\mathrm{c}: 2] ! @[\mathrm{c}: 3]([\mathrm{cHO}])[\mathrm{nX} 2 \mathrm{HO} 0: 4]$

20

[aH0:1][c:2]!@[OX2:3][!\#1:4]

21

[!\#1:1][CH2:2]!@[n:3][a:4]

22

[cH1:1][a:2]([cH1])!@[a:3]([sX2,o,nX2H0,nX3H1:4])([cH1])

23

[OX2:1][CX4H2:2]!@[CX4H2:3][OX2:4]

24

[O:1]=[C:2]!@[NX3H1:3]([H:4])(cn)

25

$[\mathrm{C}] \backslash[\mathrm{CX} 3: 1]([\mathrm{H}])=[\mathrm{CX} 3: 2]([\mathrm{H}]) ! @ \backslash[\mathrm{CH} 2: 3][\mathrm{C}: 4]$

26

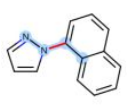

5

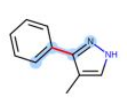

5

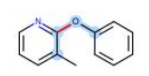

5

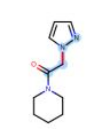

4

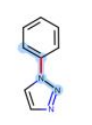

4

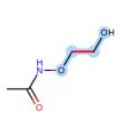

4

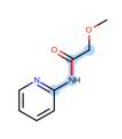

4

3 


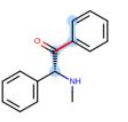

$28 \quad[\mathrm{cH} 1: 1][\mathrm{a}: 2]([\mathrm{cH} 1]) ! @[\mathrm{a}: 3]([\mathrm{cH} 1])[\mathrm{cH} 1: 4]$

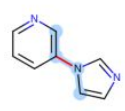

29

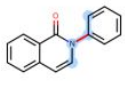

30

[cH1,nX2H0:1][c:2]([cH1,nX2H0])!@[NX3r:3][*:4]

3

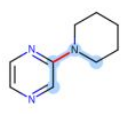

$31 \quad[\mathrm{cH0} 0: 1][\mathrm{c:2}]([\mathrm{cH} 0]) ! @[\mathrm{c:3}]([\mathrm{cH} 1: 4])[\mathrm{cH} 1]$

3

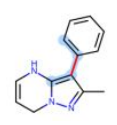

$32 \quad[\mathrm{cHO} 0: 1][\mathrm{c:2}]([\mathrm{cHO}]) ! @[0: 3][\mathrm{C}: 4]$

3<smiles>[Te]=[Te]</smiles>

$33 \quad[\mathrm{cHO} 0: 1][\mathrm{c:2}]([\mathrm{cHO}]) ! @[\mathrm{CX} 3: 3]=[0: 4]$

3

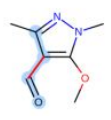

34

[a:1][c:2]([a])!@[0:3][CX4H0:4]

3

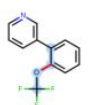

35

[a:1][c:2]!@[CX4H2:3][N,O:4]

3 
$36 \quad[\mathrm{a}: 1][\mathrm{c}: 2] ! @[\mathrm{CX} 4 \mathrm{H} 0: 3][\mathrm{CX} 3: 4]$

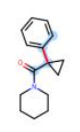

$37 \quad[0: 1][C X 4: 2] ! @[C \times 3: 3]=[0: 4]$

3

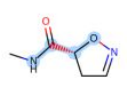

38

[O:1]=[CX3:2]!@[NX3H1:3][!\#1:4]

3

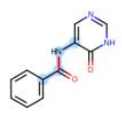

39

[O:1]=[C:2]!@[NX3H0:3]([a:4])[A]

3

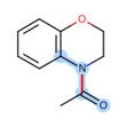

40

$[C] \backslash[C X 3: 1]([\mathrm{H}])=[C X 3: 2]([\mathrm{H}]) ! @ /[\mathrm{CH} 2: 3][\mathrm{C}: 4]$

3

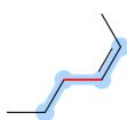

$41 \quad[\mathrm{CX} 3: 1]=[\mathrm{CX} 3: 2] ! @[\mathrm{CH} 2: 3][\mathrm{c}: 4]$

3

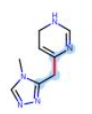

42

$[\mathrm{CX3:1]}=[\mathrm{CX} 3: 2] ! @[\mathrm{CH} 1: 3](\mathrm{C})[\mathrm{C}: 4]$

3

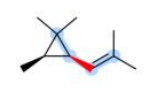

43 


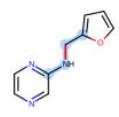

45

$\mathrm{O}[\mathrm{c}: 1][\mathrm{c}: 2](\mathrm{cO}) ! @[0: 3][\mathrm{C}: 4]$

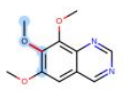

46

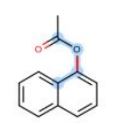

47

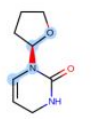

48

$[\mathrm{s}: 1][\mathrm{c}: 2] ! @[\mathrm{C}: 3]([\mathrm{NH} 1])=[\mathrm{O}: 4]$

2

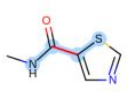

49

[nX2:1][c:2]([nX2])!@[0:3][C:4]

2

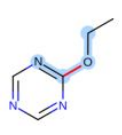

50

[cH1:1][c:2](cO)!@[0:3][C:4]

2

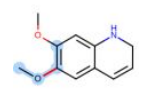

51

[cH1:1][c:2]([nX3H1])!@[NX3H1:3][C,c:4]

2

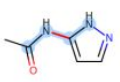

52

[cH1:1][c:2]([nX2])!@[0:3][C:4]

2 


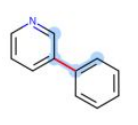

54

[cH1:1][c:2]([cH1])!@[NX3:3][CX4:4]

2

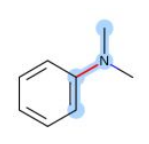

$55 \quad[\mathrm{cH} 1: 1][\mathrm{a}: 2]([\mathrm{cH} 1]) ! @[\mathrm{a}: 3]([\mathrm{sX} 2, \mathrm{o}, \mathrm{nX} 2 \mathrm{HO}, \mathrm{nX} 3 \mathrm{H} 1: 4])$

2

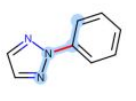

$56 \quad[\mathrm{cHO}: 1][\mathrm{c}: 2]([\mathrm{nX} 2 \mathrm{HO}]) ! @[\mathrm{NX} 3 \mathrm{H} 1: 3][\mathrm{C}, \mathrm{c}: 4]$

2

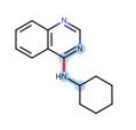

$57 \quad[\mathrm{cHO} 0: 1][\mathrm{c:2}]([\mathrm{cH} 1]) ! @[0: 3][\mathrm{C}: 4]$

2

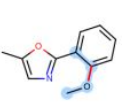

$58 \quad[\mathrm{cHO} 0: 1][\mathrm{c}: 2]([\mathrm{cH} 1]) ! @[\mathrm{NX} 3 \mathrm{H} 1: 3][\mathrm{C}, \mathrm{c}: 4]$

2

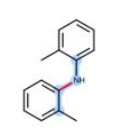

$59 \quad[\mathrm{cHO} 0: 1][\mathrm{c:2}]([\mathrm{cH} 1]) ! @[\mathrm{CX} 3: 3](\mathrm{c})=[0: 4]$

2

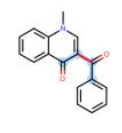

$60 \quad[\mathrm{cHO}: 1][\mathrm{c}: 2]([\mathrm{cHO}]) ! @[\mathrm{c}: 3][\mathrm{nX} 2 \mathrm{H0} 0: 4]$

2 

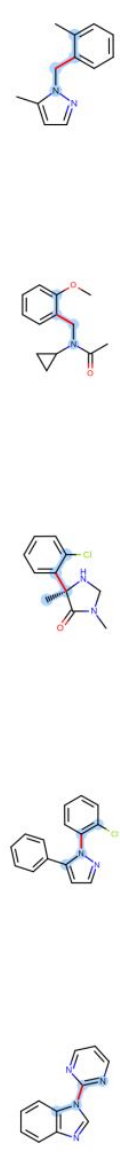


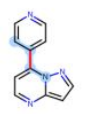

71

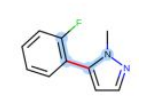

$72 \quad[\mathrm{c}: 1][\mathrm{CX} 4 \mathrm{H} 2: 2] ! @[\mathrm{OX2}: 3][\mathrm{C}: 4]$
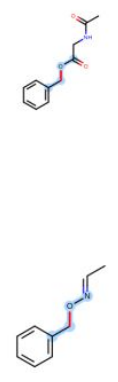

$74 \quad[\mathrm{aH} 1: 1][\mathrm{c}: 2]([\mathrm{aH} 1]) ! @[\mathrm{SX2}: 3][! \# 1: 4]$

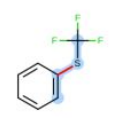

75

[a:1][c:2]!@[CX4H2:3][!\#1:4]

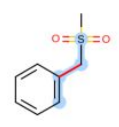

76

[a:1][c:2]!@[CX4H1:3][a:4]

2

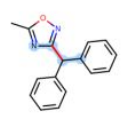

77

[a:1][c:2]!@[CX4H0:3][*:4]

2

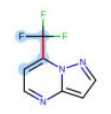




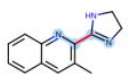

$79 \quad[a: 1][c: 2] ! @[C: 3](=[N: 4][! \# 1])(N[! \# 1])$

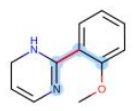

80

[a:1][c:2]!@[C:3](=[N:4])

2

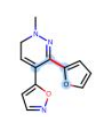

81

[a:1][ar5:2]!@[ar5:3][a:4]

2

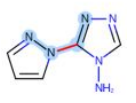

82

[a:1][a:2]!@[a:3][a:4]!@a

2

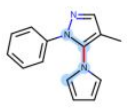

$83 \quad$ [a:1][a:2]!@[NX3:3][!\#1:4]

2

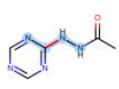

84

[a:1][a:2]!@[CX3:3]=[CX3H1:4]

2

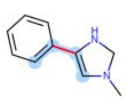

85

[OX2:1][CX4H2:2]!@[CX4H2:3][N:4]

2

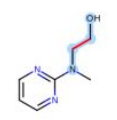

86

[OH1:1][CX4:2]!@[CX3:3]=[0:4]

2 

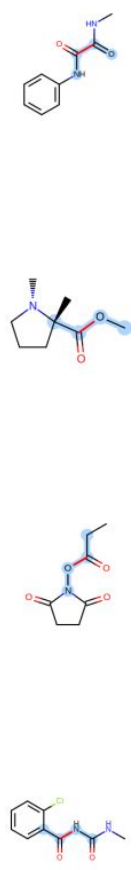

91

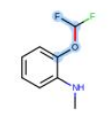

93

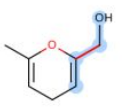




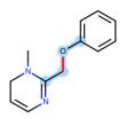

96
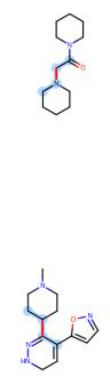

98

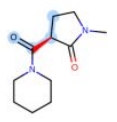

99

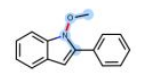

100

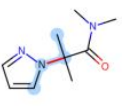

101
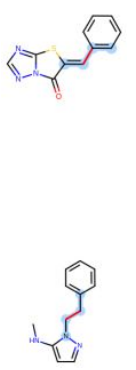

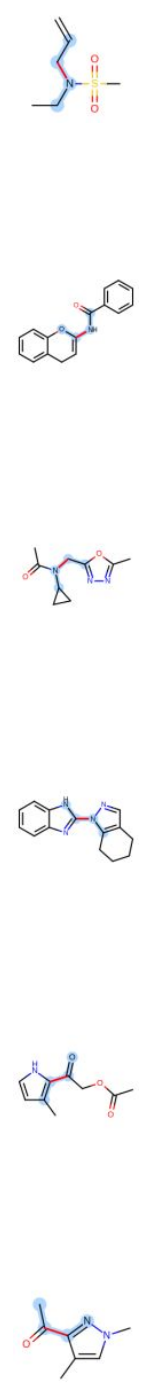


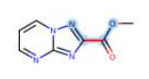

$114 \quad[n X 2: 1][c: 2] ! @[N X 2: 3]=[C: 4]([N X 3]) N$

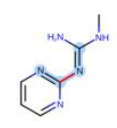

115

$[\mathrm{cH} 1: 1][\mathrm{c}: 2]([\mathrm{nX3}]) ! @[0: 3][\mathrm{C}: 4]$

1

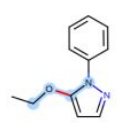

116

[cH1:1][c:2]([cH1])!@[O:3][C:4]

1

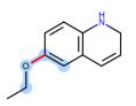

117

[cH1:1][c:2]([cH1])!@[NX3:3][a:4]

1

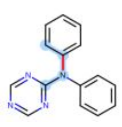

118

$[\mathrm{cH} 1: 1][\mathrm{c}: 2]([\mathrm{cH} 1]) ! @[\mathrm{CX} 3: 3](\mathrm{c})=[0: 4]$

1

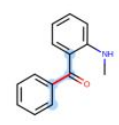

119

$[\mathrm{cH} 1: 1][\mathrm{c}: 2]([\mathrm{cH} 1]) ! @[\mathrm{CX} 3: 3]([\mathrm{CX} 3 \mathrm{HO}])=[0: 4]$

1

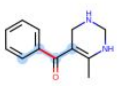

120

[cH1:1][c:2]([cH1])!@[C:3]([NH1,NH2])=[0:4]

1 


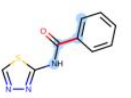

$121 \quad[\mathrm{cH} 1: 1][\mathrm{a}: 2]([\mathrm{cHO}]) ! @[\mathrm{a}: 3]([\mathrm{cHO}])[\mathrm{cHO}: 4]$

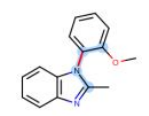

122

[cH1,n:1][c:2]!@[O:3][CRH1:4]

1

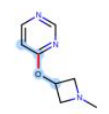

123

[cH1,n:1][c:2]!@[0:3][CH1:4]

1

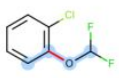

124

[cH0:1][c:2]([cH1])!@[c:3]([cHO:4])[cH1]

1

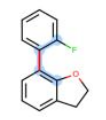

125

[cH0:1][c:2]([cH1])!@[CX3:3]=[O:4]

1

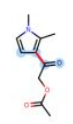

126

[cH0:1][c:2]([cHO])!@[0:3][c:4]

1

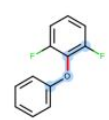

127

[cH0:1][c:2]([cHO])!@[NX3H1:3][C,c:4]

1

这

128

[cH0:1][c:2]([cHO])!@[C:3](=[N:4])

1

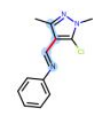



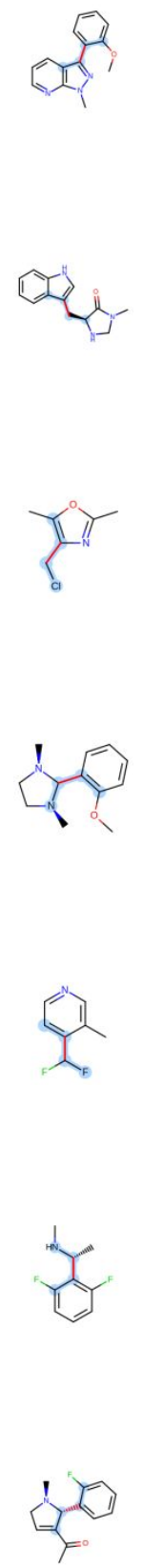


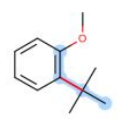

$138 \quad[\mathrm{cHO}: 1][\mathrm{c}: 2] ! @[\mathrm{CX} 4 \mathrm{HO} 0: 3][*: 4]$

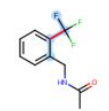

139

[cH0:1][c:2]!@[C:3](=[N:4])

1

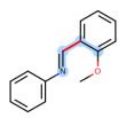

140

[cHO:1]([OHO]) $[\mathrm{c:2}]([\mathrm{cH} 1]) ! @[\mathrm{C}: 3](=\mathrm{O})[\mathrm{NH} 1: 4]$

1

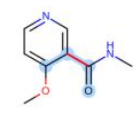

141

$[\mathrm{cHO}: 1](\mathrm{Cl})[\mathrm{c}: 2]([\mathrm{cH} 1]) ! @[\mathrm{CX} 3: 3]=[0: 4]$

1

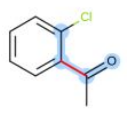

142

[c:1][CX4H2:2]!@[CX3:3]=[0:4]

1

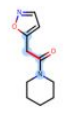

143

$[\mathrm{c}: 1]([\mathrm{NH} 1, \mathrm{NH} 2])[\mathrm{c}: 2] ! @[\mathrm{CX} 3: 3](=[\mathrm{O}: 4])([\mathrm{O}])$

1

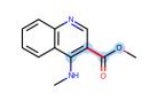

$144 \quad[\mathrm{c}: 1]([\mathrm{NH} 1, \mathrm{NH} 2])[\mathrm{c}: 2] ! @[\mathrm{CX} 3: 3](=[0: 4])([! \mathrm{O}])$

1

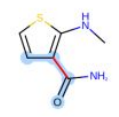

145

[aH1:1][c:2]([aHO])!@[SX2:3][!\#1:4]

1

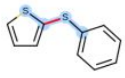




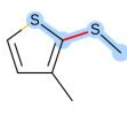

147 [a:1][c:2]!@[CX4H2:3][CX4:4]

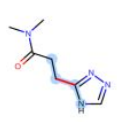

148

[a:1][c:2]!@[CX4H2:3][CX3:4]

1

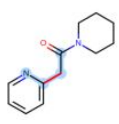

149

[a:1][c:2]!@[CX4H1:3][N,O:4]

1

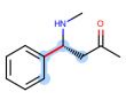

150

[a:1][c:2]!@[CX4H1:3][H:4]

1

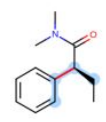

151 [a:1][c:2]!@[CX3:3]=[CX3H0:4]

1

को

152

[a:1][c:2]!@[C:3]([NH1,NH2])=[O:4]

1

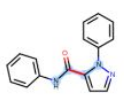

153

[a:1][c:2]!@[C:3]([NHO])=[0:4]

1

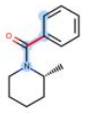




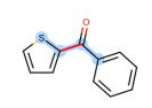

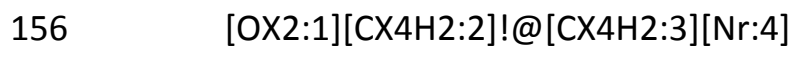

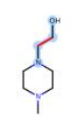

157
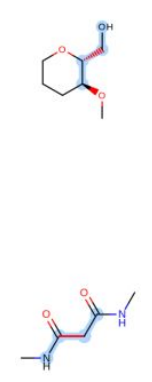

159

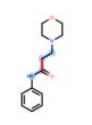

160
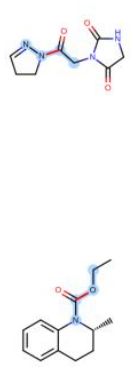


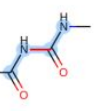

$164 \quad[\mathrm{O}: 1]=[\mathrm{C}: 2] ! @[N X 3: 3](\mathrm{C}=0)\left[{ }^{*}: 4\right]$
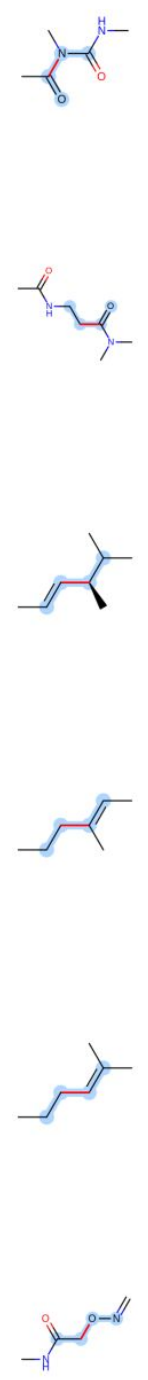

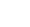

\footnotetext{
$\left[{ }^{*}: 1\right] \sim[\mathrm{CX} 3.2] ! @[N X 2: 3] \sim[*: 4]$
}

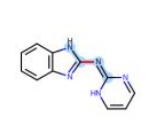




$$
\text { oyon }
$$
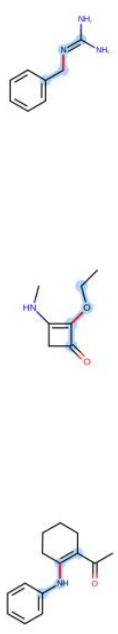

176

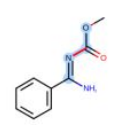

177

[*:1] [CX3:2]!@[CX3:3] [*:4]
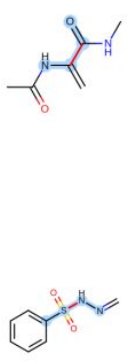


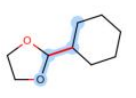

181

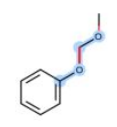

182

[!\#1:1][CX4H2:2]!@[OX2:3][C:4]

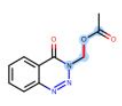

183

[!\#1:1][CX4H2:2]!@[CX3:3]=[0:4]

1

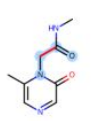

184

[!\#1:1][CX4H0:2]!@[OX2:3][!\#1:4]

1

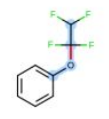

185

[!\#1:1][CX4:2]!@[SX4:3][!\#1:4]

1

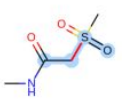

186

[!\#1:1][CX4:2]!@[NX3:3][!\#1:4]

1

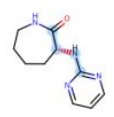

187

[!\#1:1][CX4:2]!@[CX3:3]=[0:4]

1

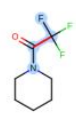




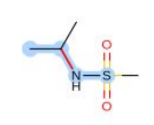

190

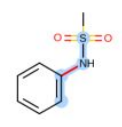

191
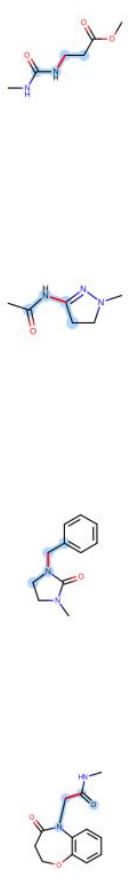

Table S2: List of torsion patterns and their frequency of occurrence in the TorsionNet 50k training set. 


\begin{tabular}{|c|c|c|}
\hline 26 & [cH1:1][c:2]([cH1])!@[NX3H1:3][C,c:4] & 467 \\
\hline 27 & [a:1][a:2]!@[NH1:3][C,c:4] & 457 \\
\hline 28 & $\mathrm{O}=[\mathrm{C}: 1][\mathrm{NX} 3 \mathrm{H} 0: 2] ! @[\mathrm{CX} 4 \mathrm{H} 1: 3][\mathrm{H}: 4]$ & 445 \\
\hline 29 & [a:1][c:2]!@[CX4H2:3][CX4:4] & 444 \\
\hline 30 & {$[\mathrm{~N}: 1][\mathrm{CX} 4: 2] ! @[\mathrm{CX} 3: 3]=[0: 4]$} & 432 \\
\hline 31 & [cH1:1][c:2]([cH1])!@[CX4H2:3][CX4:4] & 426 \\
\hline 32 & {$[\mathrm{cH0}: 1][\mathrm{c}: 2]([\mathrm{cH} 1]) ! @[0: 3][\mathrm{C}: 4]$} & 410 \\
\hline 33 & {$[*: 1] \sim[C X 4: 2] ! @[n: 3] \sim[*: 4]$} & 390 \\
\hline 34 & {$[\mathrm{cH} 1, \mathrm{nX} 2 \mathrm{HO}: 1][\mathrm{c}: 2]([\mathrm{cH} 1, \mathrm{nX} 2 \mathrm{H0}]) ! @[\mathrm{NX} 3 \mathrm{r}: 3]\left[{ }^{*}: 4\right]$} & 369 \\
\hline 35 & {$[\mathrm{c}: 1][\mathrm{c}: 2] ! @[\mathrm{c}: 3][\mathrm{s}, \mathrm{o}, \mathrm{nX} 3 \mathrm{H} 1: 4]$} & 359 \\
\hline 36 & [OX2:1][CX4:2]!@[CX4:3][OX2:4] & 356 \\
\hline 37 & {$\left[{ }^{*}: 1\right]^{\sim}[\mathrm{CX} 3: 2] ! @[\mathrm{NX} 3: 3]^{\sim}[*: 4]$} & 355 \\
\hline 38 & [NH1:1][CX4:2]!@[CX3:3]=[0:4] & 354 \\
\hline 39 & {$[\mathrm{cH} 1: 1][\mathrm{c}: 2]([\mathrm{cH} 1]) ! @[0: 3][\mathrm{C}: 4]$} & 350 \\
\hline 40 & [a:1][c:2]!@[C:3]([NH1,NH2])=[O:4] & 347 \\
\hline 41 & [cH0:1][c:2]!@[CX4H1:3][CX4:4] & 324 \\
\hline 42 & [cH0:1][c:2]!@[CX4H2:3][CX4:4] & 303 \\
\hline 43 & [O:1][CX4:2]!@[CX3:3]=[0:4] & 301 \\
\hline 44 & [C:1][CX4H2:2]!@[CX3:3]=[O:4] & 290 \\
\hline 45 & O=[C:1][NX3:2]!@[!\#1:3][!\#1:4] & 289 \\
\hline 46 & [a:1][c:2]!@[CX4H2:3][a:4] & 283 \\
\hline 47 & [!\#1:1][CX4:2]!@[NX3:3][!\#1:4] & 282 \\
\hline 48 & [cH0:1][c:2]([cH1])!@[NX3H1:3][C,c:4] & 282 \\
\hline 49 & $\mathrm{O}=[\mathrm{C}: 1][0: 2] ! @[\mathrm{CH} 2: 3]^{\sim}[\mathrm{C}: 4]$ & 282 \\
\hline 50 & {$[\mathrm{c}: 1][\mathrm{c}: 2] ! @[\mathrm{c}: 3][\mathrm{nX} 2 \mathrm{HO} 0: 4]$} & 271 \\
\hline
\end{tabular}




\begin{tabular}{|c|c|c|}
\hline 76 & {$[\mathrm{~N}: 1][\mathrm{C}: 2](=\mathrm{O}) ! @[\mathrm{CX} 4 \mathrm{H} 2: 3][\mathrm{CX} 4 \mathrm{H} 2: 4]$} & 145 \\
\hline 77 & {$[\mathrm{cH} 1, \mathrm{n}: 1][\mathrm{c}: 2] ! @[0: 3][\mathrm{CRH} 1: 4]$} & 145 \\
\hline 78 & {$[0: 1]=[C: 2] ! @[N X 3 H 0: 3]([a: 4])[A]$} & 139 \\
\hline 79 & 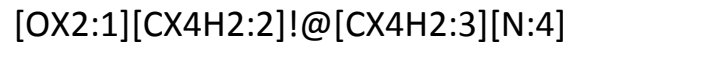 & 138 \\
\hline 80 & {$[\mathrm{O}: 1]=[\mathrm{C}: 2]([\mathrm{O}-]) ! @[\mathrm{CX} 4 \mathrm{H} 1: 3][\mathrm{H}: 4]$} & 132 \\
\hline 81 & [O:1]=[CX3:2]([NX3H1]C)!@[NX3H1:3][!\#1:4] & 128 \\
\hline 82 & {$[\mathrm{cH} 1: 1][\mathrm{c}: 2](\mathrm{cO}) ! @[0: 3][\mathrm{C}: 4]$} & 126 \\
\hline 83 & {$[\mathrm{O}: 1]=[\mathrm{C}: 2]([\mathrm{N}]) ! @[0: 3] \sim[\mathrm{C}: 4]$} & 125 \\
\hline 84 & $\mathrm{O}=[\mathrm{C}: 1][\mathrm{NX} 3 \mathrm{H} 1: 2] ! @[\mathrm{CX} 4 \mathrm{H} 0: 3][\mathrm{C}: 4]$ & 120 \\
\hline 85 & {$[\mathrm{cHO}: 1][\mathrm{c}: 2] ! @[\mathrm{CX} 4 \mathrm{HO} 0: 3][*: 4]$} & 118 \\
\hline 86 & [cH0:1][c:2]!@[CX4H2:3][a:4] & 113 \\
\hline 87 & [a:1][c:2]!@[CX4H2:3][CX3:4] & 113 \\
\hline 88 & [aH0:1][c:2]!@[OX2:3][!\#1:4] & 112 \\
\hline 89 & {$[\mathrm{cH} 1: 1][\mathrm{c}: 2]([\mathrm{cH} 1]) ! @[0: 3][\mathrm{c}: 4]$} & 112 \\
\hline 90 & [a:1][c:2]!@[C:3](=[N:4]) & 109 \\
\hline 91 & [a:1][c:2]!@[CX3:3]=[CX3H0:4] & 109 \\
\hline 92 & [aH0:1][c:2]([aHO])!@[SX2:3][!\#1:4] & 107 \\
\hline 93 & {$[\mathrm{cH} 1: 1][\mathrm{c}: 2]([\mathrm{cHO}]) ! @[0: 3][\mathrm{c}: 4]$} & 107 \\
\hline 94 & [O:1]=[C:2]!@[O:3] [CHO:4] & 103 \\
\hline 95 & {$[\mathrm{cH} 1: 1][\mathrm{c}: 2]([\mathrm{cH} 1]) ! @[\mathrm{CX} 3: 3]=[0: 4]$} & 101 \\
\hline 96 & [a:1][a:2]!@[CX3:3]=[0:4] & 99 \\
\hline 97 & [c:1][CX4:2]!@[CX3:3]=[0:4] & 97 \\
\hline 98 & $\mathrm{O}=[\mathrm{C}: 1][\mathrm{O}: 2] ! @[\mathrm{CH} 1: 3][\mathrm{H}: 4]$ & 96 \\
\hline 99 & {$[\mathrm{c}: 1][\mathrm{c}: 2] ! @[\mathrm{c}: 3]([\mathrm{cHO}])[\mathrm{nX} 2 \mathrm{HO}: 4]$} & 96 \\
\hline 100 & [CX3:1]=[CX3:2]!@[CH2:3][!\#1:4] & 6 \\
\hline
\end{tabular}




\begin{tabular}{|c|c|}
\hline 101 & [a:1][c:2]!@[CX4H0:3][a:4] \\
\hline 102 & [aH1:1][c:2]([aH1])!@[SX4:3][!\#1:4] \\
\hline 103 & [OX2:1][CX4H2:2]!@[CX4H2:3][Nr:4] \\
\hline 104 & {$[\mathrm{cH} 1, \mathrm{n}: 1][\mathrm{c}: 2] ! @[0: 3][\mathrm{CH} 1: 4]$} \\
\hline 105 & [!\#1:1][CX4:2]!@[CX3H0:3][!\#1:4] \\
\hline 106 & {$[\mathrm{cHO}: 1][\mathrm{c}: 2]([\mathrm{cHO}]) ! @[0: 3][\mathrm{C}: 4]$} \\
\hline 107 & {$[\mathrm{cHO}: 1][\mathrm{c}: 2]([\mathrm{cH} 1]) ! @[\mathrm{CX} 3: 3]=[0: 4]$} \\
\hline 108 & [!\#1:1][CX4H0:2]!@[OX2:3][!\#1:4] \\
\hline 109 & [a:1][c:2]!@[CX4H1:3][a:4] \\
\hline 110 & [O:1]=[CX3:2]!@[CX4H1r3:3][H:4] \\
\hline 111 & [OH1:1][CX4:2]!@[CX3:3]=[O:4] \\
\hline 112 & {$[\mathrm{cH} 1: 1][\mathrm{a}: 2]([\mathrm{cH} 1]) ! @[\mathrm{a}: 3]([\mathrm{sX} 2, \mathrm{o}, \mathrm{nX} 2 \mathrm{HO}, \mathrm{nX} 3 \mathrm{H} 1: 4])([\mathrm{cHO}])$} \\
\hline 113 & {$[\mathrm{cHO}: 1][\mathrm{c}: 2]([\mathrm{cHO}]) ! @[\mathrm{NX} 3 \mathrm{H} 1: 3][\mathrm{C}, \mathrm{c}: 4]$} \\
\hline 114 & {$[\mathrm{cH} 1: 1][\mathrm{c}: 2]([\mathrm{nX} 2]) ! @[0: 3][\mathrm{C}: 4]$} \\
\hline 115 & [aH0:1][c:2]([aH1])!@[SX4:3][!\#1:4] \\
\hline 116 & {$[\mathrm{c}: 1]([\mathrm{NH} 1, \mathrm{NH} 2])[\mathrm{c}: 2] ! @[\mathrm{CX} 3: 3](=[\mathrm{O}: 4])([! \mathrm{O}])$} \\
\hline 117 & {$[\mathrm{cH} 1: 1][\mathrm{c}: 2]([\mathrm{cH} 1]) ! @[\mathrm{c}: 3]([\mathrm{cH} 1: 4])[\mathrm{cH} 1]$} \\
\hline 118 & [nX2H0:1][c:2]!@[c:3][nX2H0:4] \\
\hline 119 & [cH0:1][c:2]!@[CX4H2:3][CX3:4] \\
\hline 120 & {$[\mathrm{a}: 1][\mathrm{c}: 2] ! @[\mathrm{CX} 4 \mathrm{HO} 0: 3][*: 4]$} \\
\hline 121 & {$[*: 1] \sim[c X 3: 2] ! @[N X 2: 3]^{\sim}[*: 4]$} \\
\hline 122 & {$[0: 1]=[C: 2] ! @[N X 3: 3](C=0)[*: 4]$} \\
\hline 123 & {$[*: 1] \sim[N X 3: 2] ! @[N X 2: 3] \sim[*: 4]$} \\
\hline 124 & {$[\mathrm{cHO}: 1][\mathrm{c}: 2]([\mathrm{nX} 2]) ! @[0: 3][\mathrm{C}: 4]$} \\
\hline 125 & [a:1][c:2]!@[CX4H2:3][!\#1:4] \\
\hline
\end{tabular}




\begin{tabular}{|c|c|}
\hline 176 & [!\#1:1][CX3:2]!@[SX2:3][!\#1:4] \\
\hline 177 & {$[\mathrm{cHO} 0: 1][\mathrm{c}: 2] ! @[\mathrm{CX} 4 \mathrm{HO} 0: 3][\mathrm{N}, \mathrm{O}, \mathrm{S}: 4]$} \\
\hline 178 & [cH0:1][c:2]!@[CX4H1:3][CX3:4] \\
\hline 179 & {$[\mathrm{~s}: 1][\mathrm{c}: 2] ! @[\mathrm{C}: 3]([\mathrm{NH} 1])=[0: 4]$} \\
\hline 180 & [O:1]=[CX3:2]!@[nX3:3][aH1:4] \\
\hline 181 & [a:1][c:2]!@[C:3](=[N:4][CX4])(N[CX4]) \\
\hline 182 & [a:1][a:2]!@[CX3:3](a)=[0:4] \\
\hline 183 & [nX3H1:1][a:2]!@[CX3:3]=[0:4] \\
\hline 184 & {$[0: 1]=[C X 3: 2] ! @[C X 3: 3]=[0: 4]$} \\
\hline 185 & {$[n X 2: 1][c: 2]([n X 2]) ! @[0: 3][C: 4]$} \\
\hline 186 & {$[\mathrm{cH0}: 1]([\mathrm{OHO}])[\mathrm{c}: 2]([\mathrm{cH} 1]) ! @[\mathrm{C}: 3](=\mathrm{O})[\mathrm{NH} 1: 4]$} \\
\hline 187 & {$[\mathrm{a}: 1]([\mathrm{NH} 1, \mathrm{NH} 2])[\mathrm{a}: 2] ! @[\mathrm{CX} 3: 3](=[\mathrm{O}: 4])([\mathrm{NX} 3 \mathrm{H} 0, \mathrm{CX} 4 \mathrm{H} 0, \mathrm{c}])$} \\
\hline 188 & [cH0:1][c:2]!@[CX4H0:3][CX4:4] \\
\hline 189 & {$[\mathrm{cH} 1: 1][\mathrm{c}: 2]([\mathrm{cH} 1]) ! @[\mathrm{NX3}: 3][\mathrm{CX} 4: 4]$} \\
\hline 190 & {$[\mathrm{c}: 1]([\mathrm{NH} 1, \mathrm{NH} 2])[\mathrm{c}: 2] ! @[\mathrm{CX} 3: 3](=[0: 4])([\mathrm{O}])$} \\
\hline 191 & [a:1][c:2]([a])!@[O:3][CX4H0:4] \\
\hline 192 & {$[! \# 1: 1][C X 4: 2] ! @[C X 3: 3]=[0: 4]$} \\
\hline 193 & {$[\mathrm{cH0}: 1][\mathrm{c}: 2]([\mathrm{cH} 0]) ! @[\mathrm{NX} 3: 3][\mathrm{CX} 4: 4]$} \\
\hline 194 & [aH0:1][c:2]([aHO])!@[SX4:3][!\#1:4] \\
\hline 195 & {$[\mathrm{cH0} 0: 1][\mathrm{c}: 2]([\mathrm{cH} 1]) ! @[\mathrm{CX} 3: 3](\mathrm{c})=[0: 4]$} \\
\hline 196 & {$[\mathrm{O}: 1]=[\mathrm{C}: 2]([\mathrm{O}-]) ! @[\mathrm{c}: 3][\mathrm{nX} 2 \mathrm{HO}: 4]$} \\
\hline 197 & {$[\mathrm{c}: 1]([\mathrm{OH} 1])[\mathrm{c}: 2] ! @[\mathrm{CX} 3: 3](=[0: 4])([! O])$} \\
\hline 198 & {$[*: 1][C X 4: 2] ! @[0: 3][C X 3: 4](=[! O])$} \\
\hline 199 & {$[C: 1][C X 4: 2] ! @[C X 3: 3][C: 4]$} \\
\hline 200 & 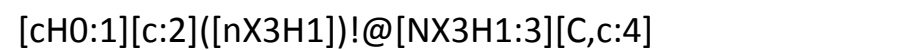 \\
\hline
\end{tabular}




\begin{tabular}{|c|c|}
\hline 201 & $\mathrm{O}=[\mathrm{C}: 1][\mathrm{NX} 3 \mathrm{HO} 0: 2] ! @[\mathrm{CX} 4 \mathrm{HO} 0: 3][\mathrm{C}: 4]$ \\
\hline 202 & {$[\mathrm{cH} 1: 1][\mathrm{c}: 2]([\mathrm{cH} 1]) ! @[\mathrm{CX} 4 \mathrm{H} 2: 3][\mathrm{CX} 4 \mathrm{H} 1: 4] \mathrm{C}(=\mathrm{O})(\mathrm{O})$} \\
\hline 203 & 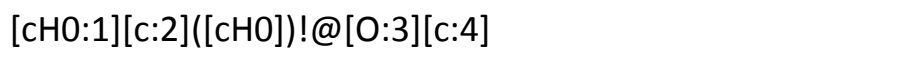 \\
\hline 204 & {$[*: 1] \sim[c X 3: 2] ! @[P: 3]^{\sim *}[4]$} \\
\hline 205 & {$[\mathrm{cHO}: 1][\mathrm{c}: 2]([\mathrm{cHO}]) ! @[\mathrm{c}: 3][\mathrm{nX} 2 \mathrm{HO}: 4]$} \\
\hline 206 & {$[\mathrm{O}: 1]=[\mathrm{C}: 2]([\mathrm{O}-]) ! @[\mathrm{c}: 3]([\mathrm{cH} 1])[\mathrm{cH} 1: 4]$} \\
\hline 207 & $\mathrm{O}=[\mathrm{S}: 1](=\mathrm{O})[\mathrm{NX} 3 \mathrm{H} 1: 2] ! @[\mathrm{CX} 4 \mathrm{H} 1: 3][\mathrm{H}: 4]$ \\
\hline 208 & [nX2H0:1][c:2](a(a)(a))!@[c:3][nX2H0:4] \\
\hline 209 & [O:1]=[C:2]!@[NX3H1:3]([H:4])(c([nX2H0])([nX2H0])) \\
\hline 210 & {$[\mathrm{cH} 1: 1][a: 2]([\mathrm{cH} 1]) ! @[a: 3]([\mathrm{sX} 2, \mathrm{o}, \mathrm{nX} 2 \mathrm{H} 0, \mathrm{nX} 3 \mathrm{H} 1: 4])([\mathrm{cH} 1])$} \\
\hline 211 & [O:1]=[C:2]([O-])!@[c:3][a:4] \\
\hline 212 & {$[c: 1][S: 2](=0)(=0) ! @[N X 3 H 0: 3][C: 4]$} \\
\hline 213 & [nX2H0:1][c:2]([nX3H1])!@[NX3H1:3][C,c:4] \\
\hline 214 & [cH1:1][a:2]([cHO])!@[a:3]([cHO])[cHO:4] \\
\hline 215 & {$[\mathrm{CX} 3: 1]=[\mathrm{CX} 3: 2] ! @[\mathrm{CH} 1: 3](\mathrm{C})[\mathrm{C}: 4]$} \\
\hline 216 & [cH1:1][c:2]([nX3H1])!@[NX3H1:3][C,c:4] \\
\hline 217 & {$[\mathrm{cHO}: 1](\mathrm{Cl})[\mathrm{c}: 2]([\mathrm{cH} 1]) ! @[\mathrm{CX} 3: 3]([\mathrm{NX} 3 \mathrm{H} 1])=[0: 4]$} \\
\hline 218 & [cH0:1][c:2]!@[CX4H1:3][N,O,S:4] \\
\hline 219 & {$[\mathrm{cH} 1: 1][\mathrm{c}: 2]([\mathrm{nX} 3]) ! @[0: 3][\mathrm{C}: 4]$} \\
\hline 220 & [a:1]([OH1])[a:2]!@[CX3:3](=[O:4])([NX3H0,CX4H0,c]) \\
\hline 221 & {$[\mathrm{C}: 1][\mathrm{S}: 2](=\mathrm{O})(=0) ! @[\mathrm{NX} 3 \mathrm{H} 1: 3][\mathrm{c}: 4]$} \\
\hline 222 & {$[*: 1] \sim[C X 4: 2] ! @[N X 2: 3]^{\sim}[*: 4]$} \\
\hline 223 & [cH0:1][c:2]!@[CX4H0:3][a:4] \\
\hline 224 & {$[\mathrm{cH} 1: 1][\mathrm{c}: 2]([\mathrm{cH} 1]) ! @[\mathrm{CX} 3: 3]([\mathrm{CX} 3 \mathrm{H} 0])=[0: 4]$} \\
\hline 225 & {$[\mathrm{nX} 2 \mathrm{HO} 0: 1][\mathrm{c}: 2](\mathrm{c}) ! @[\mathrm{CX} 3: 3]([! \mathrm{O}])=[0: 4]$} \\
\hline
\end{tabular}




\begin{tabular}{|c|c|}
\hline 226 & {$[*: 1][S: 2](=0)(=0) ! @[N X 3 H 1: 3][*: 4]$} \\
\hline 227 & [c:1][CX4H2:2]!@[OX2:3][!\#1:4] \\
\hline 228 & {$[\mathrm{cHO}: 1](\mathrm{F})[\mathrm{c}: 2]([\mathrm{cH} 1]) ! @[\mathrm{CX} 3: 3]([\mathrm{NX} 3 \mathrm{H} 1])=[\mathrm{O}: 4]$} \\
\hline 229 & {$[*: 1]^{\sim}[\mathrm{CX} 4: 2] ! @[\mathrm{CX} 3: 3]^{\sim}\left[\left[^{*}: 4\right]\right.$} \\
\hline 230 & {$[! \# 1: 1][C X 3: 2](=S) ! @[N X 3 H 0: 3][! \# 1: 4]$} \\
\hline 231 & {$[\mathrm{NH} 2][\mathrm{C}: 1](=[\mathrm{NH} 2])[\mathrm{NH}: 2] ! @[\mathrm{CH} 2: 3][\mathrm{C}: 4]$} \\
\hline 232 & [nX3H1:1][a:2]!@[a:3][o,s,nX2H0:4] \\
\hline 233 & {$[*: 1]^{\sim}[\mathrm{NX2}: 2] ! @[n X 3: 3]^{\sim}[*: 4]$} \\
\hline 234 & [0:1][CX4:2]!@[0:3][CX4:4] \\
\hline 235 & {$[*: 1]^{\sim}[n X 3: 2] ! @[O X 2: 3]^{\sim}\left[*^{*}: 4\right]$} \\
\hline 236 & {$[\mathrm{c}: 1]([\mathrm{OH} 1])[\mathrm{c}: 2] ! @[\mathrm{CX} 3: 3](=[0: 4])([\mathrm{O}])$} \\
\hline 237 & {$[\mathrm{C}: 1][\mathrm{NH}: 2] ! @[\mathrm{C}: 3](=[\mathrm{NH} 2: 4])[\mathrm{NH} 2]$} \\
\hline 238 & [c:1][cr6:2]!@[cr6:3][c:4] \\
\hline 239 & $\mathrm{O}=[\mathrm{S}: 1](=\mathrm{O})[\mathrm{NX} 3 \mathrm{H} 0: 2] ! @[\mathrm{CX} 4 \mathrm{H} 1: 3][\mathrm{H}: 4]$ \\
\hline 240 & $\mathrm{O}=[\mathrm{C}: 1][\mathrm{NX} 3 \mathrm{H} 1: 2] ! @[\mathrm{CX} 3: 3]=\left[{ }^{*} \mathrm{H} 1: 4\right]$ \\
\hline 241 & {$[\mathrm{O}-: 1][\mathrm{N}+: 2](=\mathrm{O}) ! @[\mathrm{c}: 3]([\mathrm{cHO}])[\mathrm{cHO}: 4]$} \\
\hline 242 & [cH0:1][a:2]([cHO])!@[a:3]([sX2,o,nX2H0,nX3H1:4])([cHO]) \\
\hline 243 & {$\left[{ }^{*}: 1\right]^{\sim}[\mathrm{CX} 4: 2] ! @[\mathrm{NX3}: 3]^{\sim}[*: 4]$} \\
\hline 244 & 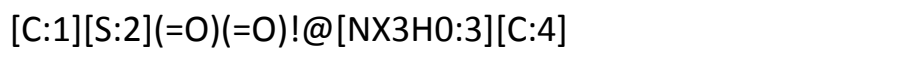 \\
\hline 245 & $\mathrm{O}=[\mathrm{S}: 1](=\mathrm{O})[\mathrm{NH} 1: 2] ! @[\mathrm{c}: 3]([\mathrm{cH} 1])[\mathrm{cH} 1: 4]$ \\
\hline 246 & [O:1]=[C:2]([O-])!@[c:3][nX3H1:4] \\
\hline 247 & [cH1:1][c:2]([cH1])!@[O:3][!C;!H:4] \\
\hline 248 & {$[*: 1][S: 2](=0)(=0) ! @[N X 3 H 0: 3][*: 4]$} \\
\hline 249 & [CX3:1]=[CX3:2]!@[CH2:3][c:4] \\
\hline 250 & {$[*: 1]^{*}[0 \times 2: 2] ! @[S X 4: 3]^{\sim}\left[\left[^{*} 4\right]\right.$} \\
\hline
\end{tabular}




\begin{tabular}{|c|c|}
\hline 251 & {$[\mathrm{cHO}: 1][\mathrm{c}: 2]([\mathrm{cHO}]) ! @[\mathrm{c}: 3]([\mathrm{cHO}: 4])[\mathrm{cH} 1]$} \\
\hline 252 & [a:1][a:2]!@[CX3:3]=[CX3H2:4] \\
\hline 253 & $\mathrm{O}=[\mathrm{C}: 1][\mathrm{NX} 3 \mathrm{H} 1: 2] ! @[\mathrm{CX} 3: 3]=\left[{ }^{*} \mathrm{H} 2: 4\right]$ \\
\hline 254 & {$[\mathrm{cHO}: 1][\mathrm{c}: 2]([\mathrm{cHO}]) ! @[\mathrm{C}: 3](=[\mathrm{N}: 4])$} \\
\hline 255 & $\mathrm{O}=[\mathrm{S}: 1](=\mathrm{O})[\mathrm{NH} 1: 2] ! @[\mathrm{c:3}]([\mathrm{cH} 1])[\mathrm{cHO}: 4]$ \\
\hline 256 & [CX3:1]=[CX3:2]!@[CX3:3]=[CX3:4] \\
\hline 257 & {$[\mathrm{cH}: 1][\mathrm{c}: 2]([\mathrm{cH}]) ! @[0: 3][\mathrm{C}: 4](\mathrm{F})(\mathrm{F})[\mathrm{F}]$} \\
\hline 258 & {$\left[{ }^{*}: 1\right]^{\sim}[\mathrm{CX} 3: 2] ! @[c X 3: 3]^{\sim}\left[{ }^{*}: 4\right]$} \\
\hline 259 & [cH1:1][a:2]([cH1])!@[a:3]([sX2,o,nX2H0,nX3H1:4]) \\
\hline 260 & {$[\mathrm{C}] \backslash[\mathrm{CX} 3: 1]([\mathrm{H}])=[\mathrm{CX} 3: 2]([\mathrm{H}]) ! @ /[\mathrm{CH} 2: 3][\mathrm{C}: 4]$} \\
\hline 261 & 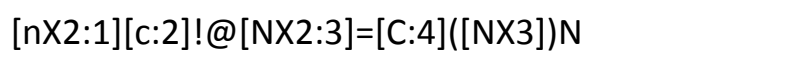 \\
\hline 262 & {$[C] \backslash[C X 3: 1]([\mathrm{H}])=[\mathrm{CX} 3: 2]([\mathrm{H}]) ! @ \backslash[\mathrm{CH} 1: 3](\mathrm{C})[\mathrm{C}: 4]$} \\
\hline 263 & [O-:1][N+:2](=O)!@[c:3][a:4] \\
\hline 264 & {$[0: 1]=[C: 2]([N H 1]) ! @[N X 3 H 1: 3](C=O)[H: 4]$} \\
\hline 265 & [a:1][ar5:2]!@[ar5:3][a:4] \\
\hline 266 & $\mathrm{O}[\mathrm{c}: 1][\mathrm{c}: 2](\mathrm{cO}) ! @[0: 3][\mathrm{C}: 4]$ \\
\hline 267 & {$[*: 1]^{\sim}[N, n: 2] ! @[P: 3] \sim[*: 4]$} \\
\hline 268 & {$[\mathrm{cHO}: 1](\mathrm{Cl})[\mathrm{c}: 2]([\mathrm{cH} 1]) ! @[\mathrm{CX} 3: 3]=[0: 4]$} \\
\hline 269 & [cH1:1][a:2]([cHO])!@[a:3]([cH1])[cHO:4] \\
\hline 270 & {$[\mathrm{C}] \backslash[\mathrm{CX} 3: 1]([\mathrm{H}])=[\mathrm{CX} 3: 2]([\mathrm{H}]) ! @ \backslash[\mathrm{CH} 2: 3][\mathrm{C}: 4]$} \\
\hline 271 & [cH0:1][c:2]!@[CX4H1:3][H:4] \\
\hline 272 & [cH0:1][a:2]([cHO])!@[a:3]([sX2,o,nX2H0,nX3H1:4]) \\
\hline 273 & {$\left[{ }^{*}: 1\right] \sim[N X 3: 2] ! @[N X 3: 3]^{\sim}\left[{ }^{*}: 4\right]$} \\
\hline 274 & {$\left[{ }^{*}: 1\right]^{\sim}[\mathrm{CX} 3: 2] ! @[N X 4: 3]^{\sim}\left[{ }^{*}: 4\right]$} \\
\hline 275 & {$[\mathrm{cHO} 0: 1][\mathrm{c}: 2] ! @[\mathrm{CX} 4 \mathrm{HO} 0: 3][\mathrm{CX} 3: 4]$} \\
\hline
\end{tabular}




\begin{tabular}{|c|c|}
\hline 276 & {$[\mathrm{O}: 1]=[\mathrm{C}: 2]([\mathrm{O}-]) ! @[\mathrm{c}: 3]([\mathrm{cH} 1])[\mathrm{cHO}: 4]([\mathrm{NH} 1, \mathrm{NH} 2])$} \\
\hline 277 & [nX2H0:1][c:2]!@[C:3](=[N:4])(-[NH1,NH2]) \\
\hline 278 & $\mathrm{O}=[\mathrm{C}: 1][\mathrm{NX} 3 \mathrm{H} 0: 2] ! @[\mathrm{CX} 3: 3]=[* \mathrm{HO} 0: 4]$ \\
\hline 279 & {$[\mathrm{cHO}: 1](\mathrm{F})[\mathrm{c}: 2]([\mathrm{cH} 1]) ! @[\mathrm{CX} 3: 3]=[0: 4]$} \\
\hline 280 & {$[\mathrm{O}: 1]=[\mathrm{C}: 2]([! \mathrm{NH} 1]) ! @[\mathrm{NX} 3 \mathrm{H} 1: 3]([\mathrm{H}: 4])(\mathrm{c}([\mathrm{nX} 2 \mathrm{HO}])([\mathrm{nX} 2 \mathrm{HO}]))$} \\
\hline 281 & [cH1:1][a:2]([cH1])!@[a:3]([cH1])[cH1:4] \\
\hline 282 & {$[\mathrm{C}: 1][\mathrm{S}: 2](=\mathrm{O})(=\mathrm{O}) ! @[\mathrm{NX} 3 \mathrm{HO} 0: 3][\mathrm{c}: 4]$} \\
\hline 283 & [nX2H0:1][a:2]([nX2H0])!@[CX3:3]=[O:4] \\
\hline 284 & [C:1][CX4:2]!@[CX3:3][c:4] \\
\hline 285 & [!\#1:1][c:2]!@[SX3:3][!\#1:4] \\
\hline 286 & {$[\mathrm{C}] \backslash[\mathrm{CX} 3: 1]([\mathrm{H}])=[\mathrm{CX} 3: 2]([\mathrm{C}]) ! @ \backslash[\mathrm{CH} 2: 3][\mathrm{C}: 4]$} \\
\hline 287 & $\mathrm{O}=[\mathrm{C}: 1][\mathrm{NX} 3 \mathrm{H} 0: 2] ! @[\mathrm{CX} 3: 3]=\left[{ }^{*} \mathrm{H} 1: 4\right]$ \\
\hline 288 & [!\#1:1][CX4H2:2]!@[OX2:3][C:4] \\
\hline 289 & 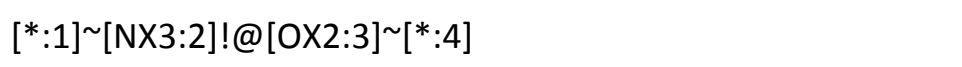 \\
\hline 290 & {$[\mathrm{O}: 1]=[\mathrm{CX} 3: 2]([\mathrm{NH} 1]) ! @[\mathrm{CH} 2: 3][\mathrm{CX} 3: 4]=0$} \\
\hline 291 & [cH0:1][a:2]([cH1])!@[a:3]([sX2,o,nX2H0,nX3H1:4]) \\
\hline 292 & $\mathrm{O}=[\mathrm{S}: 1](=\mathrm{O})[\mathrm{NH} 0: 2] ! @[\mathrm{c}: 3]([\mathrm{cH} 1])[\mathrm{cH} 1: 4]$ \\
\hline 293 & [cH0:1][a:2]([cH0])!@[a:3]([sX2,o,nX2H0,nX3H1:4])([cH1]) \\
\hline 294 & {$[\mathrm{cH} 1: 1][\mathrm{c}: 2]([\mathrm{cH} 1]) ! @[0: 3][\mathrm{S}: 4]$} \\
\hline 295 & [cH1:1][c:2]([cH0])!@[NX3:3][a:4] \\
\hline 296 & $\mathrm{O}=[\mathrm{C}: 1][\mathrm{NX} 3 \mathrm{H} 1: 2] ! @[\mathrm{CX} 3: 3]=\left[{ }^{*} \mathrm{H} 0: 4\right]$ \\
\hline 297 & {$\left[{ }^{*}: 1\right]^{\sim}[\mathrm{NX3:2}] ! @[n X 3: 3]^{\sim}\left[{ }^{*}: 4\right]$} \\
\hline 298 & {$[*: 1]^{\sim}[\mathrm{cX3}: 2] ! @[N X 4: 3]^{\sim}[*: 4]$} \\
\hline 299 & $\mathrm{O}=[\mathrm{S}: 1](=\mathrm{O})[\mathrm{NH} 0: 2] ! @[\mathrm{c}: 3]([\mathrm{cH} 1])[\mathrm{cHO}: 4]$ \\
\hline 300 & {$[0: 1]=[\mathrm{C}: 2]([0-]) ! @[\mathrm{c}: 3][\mathrm{a}: 4][\mathrm{CX} 3]=0$} \\
\hline
\end{tabular}




\begin{tabular}{|c|c|}
\hline 301 & [cH0:1][c:2]!@[CX4H2:3][!\#1:4] \\
\hline 302 & [!\#1:1][CX4H2:2]!@[OX2:3][c:4] \\
\hline 303 & {$[\mathrm{cHO}: 1][\mathrm{c}: 2]([\mathrm{cHO}]) ! @[\mathrm{CX} 3: 3](\mathrm{c})=[0: 4]$} \\
\hline 304 & {$[! \# 1: 1][S: 2](=0)(=0) ! @[n X 3: 3][a H 0: 4]$} \\
\hline 305 & {$[c: 1][S: 2](=0)(=0) ! @[N X 3 H 1: 3][c: 4]$} \\
\hline 306 & [CHO:1][NX3:2]([CHO])!@[c:3][a:4] \\
\hline 307 & {$[0: 1]=[C: 2]([0-]) ! @[c: 3][a: 4] C(=0)(0)$} \\
\hline 308 & $\mathrm{O}=[\mathrm{S}: 1](=0)[\mathrm{N}: 2] ! @[\mathrm{c}: 3][\mathrm{a}: 4]$ \\
\hline 309 & {$[*: 1]^{\sim}[\mathrm{CX} 3: 2] ! @[\mathrm{n}: 3]^{\sim}\left[\left[^{*}: 4\right]\right.$} \\
\hline 310 & {$[*: 1][S X 2: 2] ! @[S X 2: 3][*: 4]$} \\
\hline 311 & {$[*: 1] \sim[N X 4: 2] ! @[N X 3: 3]^{\sim}\left[\left[^{*}: 4\right]\right.$} \\
\hline 312 & [a:1][a:2]!@[a:3][a:4]!@a \\
\hline 313 & [c:1][cr5:2]!@[cr5:3][c:4] \\
\hline 314 & $\mathrm{O}=[\mathrm{S}: 1](=0)[\mathrm{NH} 1: 2] ! @[\mathrm{c}: 3][\mathrm{nX2}: 4]$ \\
\hline 315 & [cH1:1][c:2]([cH1])!@[NX3:3][a:4] \\
\hline 316 & {$[\mathrm{cH} 1: 1][\mathrm{c}: 2] ! @[N X 2: 3]=[\mathrm{C}: 4]([\mathrm{NX} 3]) \mathrm{N}$} \\
\hline 317 & [!\#1:1][CX4:2]!@[SX3:3][!\#1:4] \\
\hline 318 & [nX2:1][c:2]([nX3])!@[0:3][C:4] \\
\hline 319 & [!\#1:1] [CX3:2]!@[SX4:3] [!\#1:4] \\
\hline 320 & {$[\mathrm{cHO}: 1]([\mathrm{CX} 3])[\mathrm{c:}: 2]([\mathrm{cH} 1]) ! @[\mathrm{CX} 3: 3](\mathrm{c})=[0: 4]$} \\
\hline 321 & [a:1][a:2]!@[NX3:3][CX4H0:4] \\
\hline 322 & {$[\mathrm{cH} 1: 1][\mathrm{c}: 2]([\mathrm{cHO}]) ! @[0: 3][\mathrm{S}: 4]$} \\
\hline 323 & {$[\mathrm{cHO}: 1][\mathrm{c}: 2]([\mathrm{cHO}]) ! @[\mathrm{c}: 3]([\mathrm{cHO}: 4])[\mathrm{cHO}]$} \\
\hline 324 & [c:1][CX4:2]!@[CX3:3][c:4] \\
\hline 325 & $\left.[*: 1] \sim[N, n: 2] ! @[S: 3]^{\sim *}: 4\right]$ \\
\hline
\end{tabular}


Table S2: List of torsion patterns present in the TorsionNet500 benchmark. The definition of torsion patterns was adopted from Scharfer ${ }^{7}$. For each torsion pattern, a representative TorsionNet500 fragment along with the number of examples containing that pattern are shown.

\section{REFERENCES}

1. Behler, J.; Parrinello, M., Generalized Neural-Network Representation of High-Dimensional Potential-Energy Surfaces. Phys. Rev. Lett. 2007, 98 (14), 146401.

2. Behler, J., Constructing High-Dimensional Neural Network Potentials: A Tutorial Review. Int. J. Quantum Chem. 2015, 115 (16), 1032-1050.

3. Mills, J. E. J.; Dean, P. M., Three-Dimensional Hydrogen-Bond Geometry and Probability Information From a Crystal Survey. J. Comput. Aided Mol. Des. 1996, 10 (6), 607-622. 
4. Lovering, F.; Bikker, J.; Humblet, C., Escape from Flatland: Increasing Saturation as an Approach to Improving Clinical Success. J. Med. Chem. 2009, 52 (21), 6752-6756.

5. Wang, R.; Fu, Y.; Lai, L., A New Atom-Additive Method for Calculating Partition Coefficients. J. Chem. Inf. Comput. Sci. 1997, 37 (3), 615-621.

6. Gehlhaar, D. K.; Verkhivker, G. M.; Rejto, P. A.; Sherman, C. J.; Fogel, D. R.; Fogel, L. J.; Freer, S. T., Molecular Recognition of the Inhibitor AG-1343 by HIV-1 Protease: Conformationally Flexible Docking by Evolutionary Programming. Chem. Biol. 1995, 2 (5), 317-324.

7. Schärfer, C.; Schulz-Gasch, T.; Ehrlich, H.-C.; Guba, W.; Rarey, M.; Stahl, M., Torsion Angle Preferences in Druglike Chemical Space: A Comprehensive Guide. J. Med. Chem. 2013, 56 (5), 20162028.

8. $\quad$ Friedrich, N.-O.; Meyder, A.; de Bruyn Kops, C.; Sommer, K.; Flachsenberg, F.; Rarey, M.; Kirchmair, J., High-Quality Dataset of Protein-Bound Ligand Conformations and Its Application to Benchmarking Conformer Ensemble Generators. J. Chem. Inf. Model. 2017, 57 (3), 529-539. 